\title{
Performance of Generalized Receiver Employed by Broadband Multicarrier DS-CDMA System Using Space-Time Spreading-Assisted Transmit Diversity
}

\author{
VYACHESLAV TUZLUKOV \\ Department of Technical Maintenance of Aviation and Radio Electronic Equipment \\ Belarusian State Aviation Academy \\ 77, Uborevicha Str., 220096 Minsk \\ BELARUS
}

\begin{abstract}
In this paper the multicarrier direct-sequence code-division multiple access (MC DS-CDMA) using space-time spreading assisted transmit diversity is investigated in the context of broadband wireless communications systems constructed based on the generalized approach to signal processing in noise over frequency-selective Rayleigh fading channels. We consider the issue of parameter design for the sake of achieving high-efficiency communications in various dispersive environments. In contrast to the conventional MC DS-CDMA wireless communication system employing the time $(T)$-domain spreading only, in the present paper the broadband wireless MC DS-CDMA wireless communication schemes employ both the time $(T)$-domain and frequency $(F)$-domain spreading, i.e., employ the $T F$-domain spreading. The bit-error rate (BER) performance of the space-time spreading assisted broadband MC DS-CDMA wireless communications system is investigated for down-link transmissions associated with the single user and multiuser generalized detectors and is compared with that of the single user correlation detector and the multiuser decorrelating detector. Our study demonstrates that with appropriately selecting the system parameters, the broadband MC DS-CDMA wireless communication system using the space-time spreading assisted transmit diversity constitutes a promising downlink transmission scheme. This scheme is capable to support ubiquitous communications over diverse communication environments without the BER performance degradation.
\end{abstract}

Key-Words: - Generalized receiver, broadband system, code-division multiple-access (CDMA), frequency-domain spreading, frequency-selective fading, multicarrier direct-sequence code-division multiple-access (MC DS-CDMA), multicarrier CDMA, multicarrier detection, space-time spreading, transmit diversity.

Received: May 20, 2021. Revised: September 14, 2021. Accepted: September 23, 2021. Published: September 29, 2021.

\section{Introduction}

One of the basic requirements to broadband wireless communication systems is to support the expected high bit rates required by wireless Internet service and for delivering high-speed multimedia services. However, the achievable capacity and data rate of wireless communication systems are limited by the time varying characteristics of the dispersive fading channel. An efficient technique of combating the time-varying effects of wireless channels is to employ diversity.

In recent years, the space-time coding has received much attention as an effective transmit diversity technique used for combating fading in wireless communication systems [1]-[4]. Inspired by the space-time codes, in [5] an attractive transmit diversity scheme based on the space-time spreading has been proposed for employment in the code-division multiple-access (CDMA) wireless communication systems [6], [7]. Space-time spreading scheme designed for supporting two transmit antennas and one receive antenna has been included also in the cdma 2000 wideband CDMA (W-CDMA) standard [6]- [8]. In [4], the performance of the single-carrier CDMA wireless communication systems using space-time spreading has been investigated, when the channel is modeled either as a flat or frequency-selective Rayleigh fading channel in the absence of multiuser interference.

In the present paper, we investigate the issues of parameter design and bit-error-rate (BER) performance of the broadband multicarrier direct-sequence CDMA (MC DS-CDMA) wireless communication systems constructed on the basis of the generalized approach to signal processing in noise [9]-[11] using the space-time spreading assisted transmit diversity when communicating over frequency-selective Rayleigh fading channels. The reason to consider the broadband MC DS-CDMA wireless communication system is because it constitutes a generalized 
multiple access scheme [6], [7], [12]-[16] which exhibits a high grade of design high flexibility.

The broadband MC DS-CDMA wireless communication system possesses a range of parameters that can be adjusted to satisfy the required design trade off. Our objective in the context of parameter design is to configure the broadband MC DS-CDMA wireless communication systems to achieve the high efficiency communications in various propagation environments characterized by different grade of dispersion.

In the present paper, specifically, the synchronous downlink (from the base station to moving platform) transmission of the user signals is considered and the $B E R$ performance is evaluated for a range of parameter values. Furthermore, in contrast to the family of conventional MC DS-CDMA wireless communication systems employing the time $(T)$-domain spreading only, in this contribution, we also investigate the broadband MC DS-CDMA wireless communication system performance when employing both the $(T)$-domain and frequency $(F)$-domain spreading i.e., employing the $T F$-domain spreading.

A typical advantage to use the $T F$-domain spreading in MC DS-CDMA wireless communication systems is that the maximum number of users supported is determined by the product of the $(T)$-domain spreading factor and the $(F)$-domain spreading factor. Therefore, the broadband MC DS-CDMA wireless communication system using the $T F$-domain spreading is capable to support a significantly higher number of users than in the case to use the solely $T$ domain spreading, since in this case, the maximum number of users is supported by the $T$-domain spreading factor alone.

The performance of the space-time spreading assisted broadband MC DS-CDMA wireless communication system using the $T F$-domain spreading is investigated in conjunction with both the single user and multiuser generalized detectors and is compared with the single user correlation detector and multiuser decorrelating detector [6],[7],[9]-[11],[15]-[17]. Our study shows that a high number of users can be supported by the broadband MC DS-CDMA wireless communication system using the $T F$-spreading, without having to impose trade off in terms of the achievable diversity order.

The remainder of this paper is organized as follows. In Section II, the space-time spreading assisted broadband MC DS-CDMA wireless communication system is described and the required parameter values are investigated. In Section III, we derive the achievable $B E R$ and characterize the $B E R$ performance of the broadband MC DS-CDMA wireless communication system using the space-time spreading. In
Section IV the achievable capacity improvement of the broadband MC DS-CDMA wireless communication system using the space-time spreading and $T F$ domain spreading is discussed. Finally, some conclusions are discussed in Section V.

\section{System Model}

\subsection{Transmitter model}

The wireless communication system considered in the present paper is the orthogonal MC DS-CDMA wireless communication system and has a similar structure as discussed in [6], [7], [13], [15] using the $P \times G$ number of subcarriers, $T$ the number of transmit antennas and one receive antenna. Furthermore, in this paper, the synchronous broadband MC DSCDMA wireless communication system is investigated where the $K$ user signals are transmitted synchronously. The transmitter schematic of the $k$-th user is shown in Fig. 1 where the real-valued data symbols using the binary phase-shift keying (BPSK) modulation and real-valued spreading [5]-[7] were considered.

Figure 2 demonstrates the frequency arrangement of $M$ subcarriers. As shown in Fig.1, at the transmitter side the block of $P \times G$ data bits each having the bit duration of $T_{b}$ is the serial-to-parallel (S-P) converted to $P$ parallel subblocks. Each parallel subblock has $G$ data bits, which are space-time spread using the schemes of [5], [15] with the aid of $M$ orthogonal spreading codes $[6]$

$$
\left\{a_{k, 1}(t), a_{k, 2}(t), \ldots, a_{k, M}(t)\right\}, \quad k=1,2, \ldots, K
$$

and mapped to $T$ transmit antennas.

The symbol duration of the space-time spreading signals is $P G T_{b}$, and the discrete period of the orthogonal codes can be presented in the following form

$$
\frac{P G T_{b}}{T_{c}}=P G N,
$$

where $N=T_{b} / T_{c}$ and $T_{c}$ represents the chip duration of the orthogonal spreading codes.

The orthogonal codes take the following form

$$
a_{k, 1}(t)=\sum_{j=0}^{P G N-1} a_{k, i}[j] P_{T_{c}}\left(t-j T_{c}\right)
$$

where $a_{k, i}[j] \in\{+1,-1\}$ and obey the following relationship

$$
\sum_{l=0}^{P G N-1} a_{i, m}[l] a_{j, n}[l]=0
$$


whenever $i \neq j$ or $m \neq n$.

Furthermore, $P_{T_{c}}(t)$ represents the chip impulse waveform defined over the interval of $\left[0, T_{c}\right)$. Since the total number of orthogonal codes having a discrete period of $P G N$ is $P G N$ and since each user requires $M$ orthogonal codes for space-time spreading, the maximum number of users supported by these orthogonal codes is $P G N / M$. As seen in Fig. 1, the following space-time spreading, each space-time spreading block generates $T$ parallel signals to be mapped to the $T$ transmit antennas. For each transmit antenna, the specific $P$ space-time spreading signals generated by the $P$ space-time spreading blocks are the repeated $S$ times, so that each spacetime signal is transmitted on $n$ subcarriers.

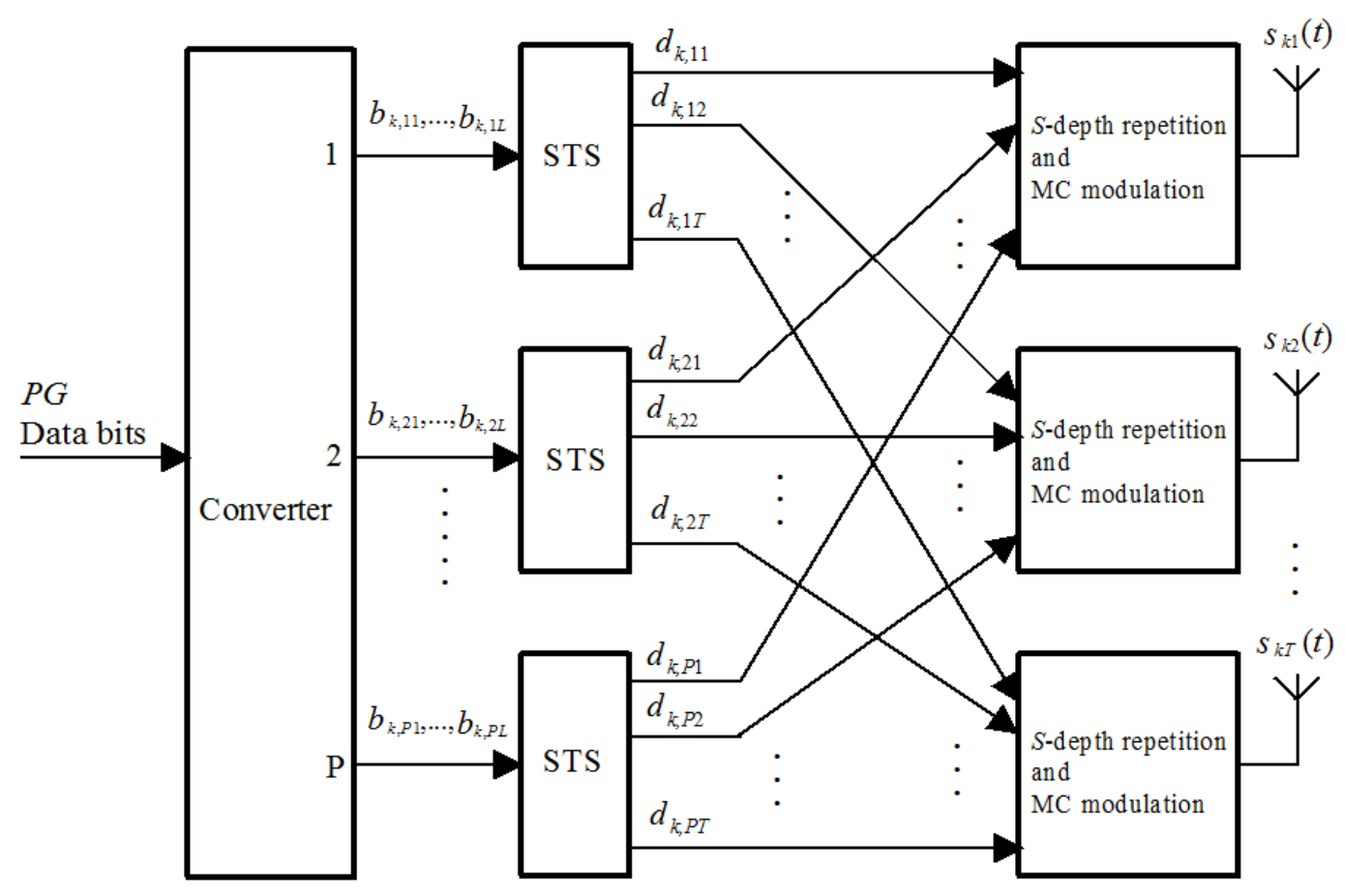

Fig.1. The transmitter schematic of the broadband MC DS-CDMA wireless communication system using space-time spreading.

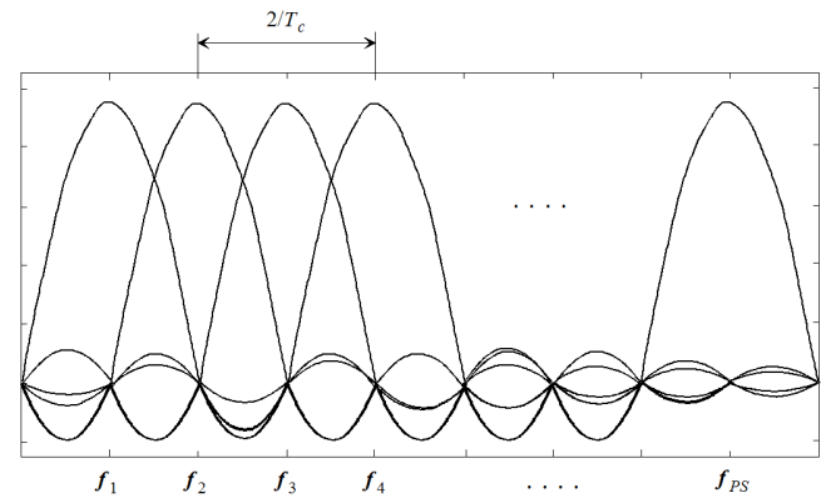

Fig. 2. Spectrum of the orthogonal broadband MC DSCDMA wireless communication system signals having a minimum subcarrier spacing of $1 / T_{c}$, where the zero-tozero bandwidth of each DS spread signal is $2 / T_{c}$.

The corresponding number $S$ of subcarriers is selected to guarantee that the same space-time spreading signal is transmitted by the specific $S$ subcarri- ers having the maximum possible frequency spacing, so that they experience independent fading and, hence, achieve the maximum frequency diversity for a given $S$ value. Specifically, let $\left\{f_{1}, f_{2}, \ldots, f_{P S}\right\}$ be the subcarrier frequencies, which are arranged according to Fig.2. These subcarrier frequencies can be written in the following matrix form

$$
\left\{f_{i}\right\}=\left(\begin{array}{lclc}
f_{1} & f_{P+1} & \cdots & f_{(S-1) P+1} \\
f_{2} & f_{P+2} & \cdots & f_{(S-1) P+2} \\
\vdots & \vdots & \ddots & \vdots \\
f_{P} & f_{2 P} & \cdots & f_{S P}
\end{array}\right) .
$$

Then, the space-time spreading signal will be transmitted using the subcarrier frequencies from the same row of (5). Finally, as shown in Fig.1, the inverse fast Fourier transform (IFFT) is invoked to carrying out multicarrier modulation, and the signal at the IFFT block output is transmitted using one of the transmit antenna. 
The general form of the $k$-th user's transmitted baseband signal corresponding to the $T$ transmit antennas can be expressed in the following form

$$
\mathbf{s}_{k}(t)=\operatorname{Re}\left\{\sqrt{\frac{2 E_{b}}{P T_{b}} \times \frac{1}{S M T}} \mathbf{D}_{k} \mathbf{P} \boldsymbol{\omega}\right\},
$$

where $E_{b} / P T_{b}$ represents the transmitted power per subcarrier expressed as

$$
\frac{G E_{b}}{P G T_{b}}=\frac{E_{b}}{P T_{b}},
$$

the factor $S$ in the denominator in (6) is due to the $S$ depth repetition, while the factor $M T$ represents the same space-time spreading using $M$ orthogonal codes and $T$ transmit antennas.

$\operatorname{In}(6)$

$$
\mathbf{s}_{k}(t)=\left[s_{k 1}(t) s_{k 2}(t) \cdots s_{k T}(t)\right]^{T},
$$

where the superscript $T$ denotes the vector or matrix transpose, represents the signal vector transmitted by $T$ antennas, $\mathbf{P}$ represents the $S$-depth repetition operation, which is a $P \times P S$ matrix expressed as

$$
\mathbf{P}=\left[\mathbf{I}_{P} \mathbf{I}_{P} \cdots \mathbf{I}_{P}\right]
$$

with $\mathbf{I}_{P}$ being the unit matrix with the rank $P$.

Furthermore, in (6), $\mathbf{D}_{k}=\left\{d_{k, i j}\right\}$ is the $P \times T$-dimensional matrix representing the output of the space-time spreading. The space-time spreading invoked in the matrix $\mathbf{D}_{k}$ can be presented in the following form

$$
\mathbf{D}_{k}=\left[\mathbf{C}_{k} \mathbf{B}_{k}\right]^{T},
$$

where $\mathbf{C}_{k}$ is the $P \times P M$-dimensional matrix constituted by orthogonal codes, which can be expressed in the following form

$$
\mathbf{C}_{k}^{T}=\left(\begin{array}{cccc}
a_{k, 1}(t) & 0 & \cdots & 0 \\
a_{k, 2}(t) & 0 & \cdots & 0 \\
\vdots & \vdots & \ddots & \vdots \\
a_{k, M}(t) & 0 & \cdots & 0 \\
0 & a_{k, 1}(t) & \cdots & 0 \\
0 & a_{k, 2}(t) & \cdots & 0 \\
\vdots & \vdots & \ddots & \vdots \\
0 & a_{k, M}(t) & \cdots & 0 \\
\vdots & \vdots & \ddots & \vdots \\
0 & 0 & \cdots & a_{k, 1}(t) \\
0 & 0 & \cdots & a_{k, 2}(t) \\
\vdots & \vdots & \ddots & \vdots \\
0 & 0 & \cdots & a_{k, M}(t)
\end{array}\right) .
$$

In (10), $\mathbf{B}_{k}$ is the $P M \times T$ matrix mapped from the $P$ subblock data bits, according to the requirements of the space-time spreading [5]. Specifically, the matrix $\mathbf{B}_{k}$ can be presented in the following form:

$$
\mathbf{B}_{k}=\left[\mathbf{B}_{k 1}^{T} \mathbf{B}_{k 2}^{T} \cdots \mathbf{B}_{k P}^{T}\right]^{T},
$$

where $\mathbf{B}_{k p}$ is the $M \times T$ dimensional matrix obeying the following structure

$$
\mathbf{B}_{k p}=\left(\begin{array}{cccc}
a_{11} b_{k, 11}^{\prime} & a_{12} b_{k, 12}^{\prime} & \cdots & a_{1 L} b_{k, 1 T}^{\prime} \\
a_{11} b_{k, 11}^{\prime} & a_{12} b_{k, 12}^{\prime} & \cdots & a_{1 L} b_{k, 1 T}^{\prime} \\
\vdots & \vdots & \ddots & \vdots \\
a_{M 1} b_{k, M 1}^{\prime} & a_{12} b_{k, M 2}^{\prime} & \cdots & a_{1 L} b_{k, M T}^{\prime}
\end{array}\right)
$$

where $p=1,2, \ldots, P ; a_{i j}$ represents the sign of the element at the $i$-th row and the $j$-th column, which is determined by the space-time spreading design rule, while $b_{k, i j}^{\prime}$ in $\mathbf{B}_{k p}$ is the data bit assigned to the $(i, j)$ th element, which is one of the $G$ input data bits $\left\{b_{k, p 1}, b_{k, p 2}, \ldots, b_{k, p G}\right\}$ of user $k$. For example, at $G=$ $M=T=2$, the corresponding $\mathbf{B}_{k p}$ matrix is given by [5]

$$
\left(\begin{array}{cc}
b_{k, p 1} & b_{k, p 2} \\
b_{k, p 2} & -b_{k, p 1}
\end{array}\right), p=1,2, \ldots, P .
$$

Finally, in (6), $\omega$ represents the multicarrier modulated vector of the length $P S$, which can be presented in the following form

$$
\boldsymbol{\omega}=\left[\exp \left(j 2 \pi f_{1} t\right), \exp \left(j 2 \pi f_{2} t\right), \ldots, \exp \left(j 2 \pi f_{P S} t\right)\right]^{T}
$$


Equation (6) represents the general form of the transmitted space-time spreading signals, regardless of the values $G, M$, and $T$. However, the investigation carried out in [5], [15] has shown that space-time spreading schemes using $G=M=T$, i.e., those having an equal number of data bits, orthogonal spacetime spreading related spreading sequences, as well as transmit antennas constitute the attractive schemes, since they are capable to prove the maximal transmit diversity without requiring extra space-time spreading codes. Therefore, in the present paper, we investigate only these attractive space-time spreading schemes, and our results are mainly based on the broadband MC DS-CDMA wireless communication systems using two or four transmit antennas.

As an example, in the case $G=M=T=2$, the signals employed by the broadband MC DS-CDMA wireless communication system and transmitted by the antenna 1 and 2 can be simply expressed in the following form:

$$
\begin{gathered}
\mathbf{s}_{k}(t)=\left(\begin{array}{c}
s_{k 1}(t) \\
s_{k 2}(t)
\end{array}\right)=\sqrt{\frac{2 E_{b}}{4_{b} P S T}} \\
\times\left(\begin{array}{l}
\sum_{p=1}^{P} \sum_{s=1}^{S}\left[a_{k, 1} b_{k, p 1}+a_{k, 2} b_{k, p 2}\right] \cos \left(2 \pi f_{(s-1) P+p} t\right) \\
\sum_{p=1}^{P} \sum_{s=1}^{S}\left[a_{k, 1} b_{k, p 1}-a_{k, 2} b_{k, p 2}\right] \cos \left(2 \pi f_{(s-1) P+p} t\right)
\end{array}\right) .
\end{gathered}
$$

Note that in (16), the explicit notation indicating the time dependence of $a_{k, i(t)}$ has been omitted for notational convenience, since in the present paper only synchronous transmissions are considered.

\subsection{Channel model and system parameter design}

The channels are assumed to be the slowly varying frequency-selective Rayleigh fading channels and the delay spreads are assumed to be limited to the range of $\left[T_{m}, T_{M}\right]$, where $T_{m}$ corresponds to the environments having the shortest delay spread considered, for example, in an indoor environment, while $T_{M}$ is associated with an environment having the highest possible delay spread, as in an urban area. Below, we impose some limitations on the set of parameters used by the space-time spreading assisted broadband MC DS-CDMA wireless communication systems, in order to ensure that the broadband $\mathrm{MC}$ DS-CDMA wireless communication systems operate efficiently in different dispersive environments having a delay spread in the range of $\left[T_{m}, T_{M}\right]$.

In order to ensure that space-time spreading maintains the required frequency diversity order in different wireless communication environments, we co- nfigure the system such that each subcarrier signal is guaranteed to experience flat fading. The required frequency diversity is attained by combining the independently faded subcarrier signals, with the aid of $F$-domain repetition. Since the delay spread experienced in different wireless communication environment is assume to be limited to the range of $\left[T_{m}, T_{M}\right]$ the flat fading condition of each subcarrier in these different wireless communication environments is satisfied, provided that $T_{c}>T_{M}$.

In order to achieve the highest possible grade of frequency diversity for a given number of combined subcarrier signals, the subcarrier signals combined must experience independent fading. This implies that the $F$-domain spacing between the specific subcarriers that are combined must be higher than the maximum coherence bandwidth of $(\Delta f)_{c M} \approx 1 / T_{m}$ [16]-[18]. Let $P$ be the number of subblocks after the $S-P$ conversion stage of Fig.1. Then, according to Fig.2 and (5), the above condition is satisfied, if $P / T_{c} \geq 1 / T_{m}$, i.e., $P \geq T_{c} / T_{m}$.

According to the above design philosophy, it can be shown that the broadband MC DS-CDMA wireless communication system having $T_{c}>T_{M}$ and $P \geq$ $T_{c} / T_{m}$ is capable to achieve a constant frequency-selective diversity order, provided that the delay spread of the wireless channels encountered falls within the limits of the interval $\left[T_{m}, T_{M}\right]$.

Assuming that $K$ user signals in the form of (6) are transmitted synchronously over the Rayleigh fading channels, the received complex low-pass equivalent signal can be expressed in the following form

$$
r(t)=\sum_{k=1}^{K} \sum_{g=1}^{T} \sqrt{\frac{2 E_{b}}{P T_{b}} \times \frac{1}{S M_{x} T}}\left(\mathbf{X}_{k}\right)_{g} \mathbf{H} \boldsymbol{\omega}+w(t),
$$

where $\left(\mathbf{X}_{k}\right)_{g}=\left(\mathbf{D}_{k} \mathbf{P}\right)_{g}$ represents the $g$-th row of the matrix $\mathbf{X} ; w(t)$ is the complex valued low-pass equivalent additive white Gaussian noise (AWGN) having a double-sided spectral density of $\mathcal{N}_{0}$, while

$$
\begin{array}{r}
\mathbf{H}=\operatorname{diag}\left\{h_{1 g} \exp \left(j \psi_{1 g}\right), h_{2 g} \exp \left(j \psi_{1 g}\right), \ldots,\right. \\
\left.h_{(P S)_{g}} \exp \left(j \psi_{(P S)_{g}}\right)\right\}, \quad g=1,2, \ldots, T
\end{array}
$$

is the diagonal matrix with the rank $P S$, which represents the channel's complex impulse response in the context of the $g$-th antenna. The coefficients $h_{i g}$, $i=1,2, \ldots, P S ; g=1,2, \ldots, T$ in the matrix $\mathbf{H}$ are independent and identically distributed (i.i.d.) random variables obeying the Rayleigh distribution law that can be presented in the following form 


$$
f_{h_{i g}}(y)=\frac{2 y}{\sigma^{2}} \exp \left(-y^{2} / \sigma^{2}\right), \quad y \geq 0
$$

where $\sigma^{2}=E\left[\left(h_{i g}\right)^{2}\right]$ and $E[\cdot]$ is the mathematical expectation. Furthermore, the phases $\psi_{i g}, i=1,2, \ldots, P S$ $g=1,2, \ldots, T$ are introduced by the fading channels and uniformly distributed within the limits of the interval $[0,2 \pi)$.

Specifically, in the case of $G=M=T=2$, the received complex low-pass equivalent signal takes the following form

$$
\begin{gathered}
r(t)=\sum_{k=1}^{K} \sum_{p=1}^{P} \sum_{s=1}^{S} \sqrt{\frac{2 E_{b}}{4 P S T_{b}}} \\
\times\left\{h_{(p s) 1} \exp \left\{j \psi_{(p s) 1}\right\}\left[a_{k, 1}(t) b_{k, p 1}+a_{k, 2}(t) b_{k, p 2}\right]\right. \\
\left.+h_{(p s) 2} \exp \left\{j \psi_{(p s) 2}\right\}\left[a_{k, 1}(t) b_{k, p 2}-a_{k, 2}(t) b_{k, p 1}\right]\right\} \\
\times \exp \left\{j 2 \pi f_{(s-1) P+p} t\right\}+w(t) .
\end{gathered}
$$

\subsection{Generalized receiver. Main functioning principles}

The generalized receiver is constructed in accordance with the generalized approach to signal processing in noise [9]-[11]. The generalized approach to signal processing in noise introduces an additional noise source that does not carry any information about the parameters of desired transmitted signal with the purpose to improve the signal processing system performance. This additional noise can be considered as the reference noise without any information about the parameters of the signal to be detected.

The jointly sufficient statistics of the mean and variance of the likelihood function is obtained under the generalized approach to signal processing in noise employment, while the classical and modern signal processing theories can deliver only a sufficient statistics of the mean or variance of the likelihood function. Thus, the generalized approach to signal processing in noise implementation allows us to obtain more information about the parameters of the desired transmitted signal incoming at the generalized receiver input. Owing to this fact, the detectors constructed based on the generalized approach to signal processing in noise technology are able to improve the signal detection performance of signal processing systems in comparison with employment of other conventional detectors.
The generalized receiver (GR) consists of three channels (see Fig.3): the GR correlation detector channel (GR CD) - the preliminary filter (PF), the multipliers 1 and 2, the model signal generator (MSG); the GR energy detector channel (GR ED) the $\mathrm{PF}$, the additional filter (AF), the multipliers 3 and 4 , the summator 1; and the GR compensation channel (GR CC) - the summators 2 and 3, the accumulator 1. The threshold apparatus (THRA) device defines the GR threshold.

As we can see from Fig.3, there are two bandpass filters, i.e. the linear systems, at the GR input, namely, the PF and AF. We assume for simplicity that these two filters or linear systems have the same amplitude-frequency characteristics or impulse responses. The AF central frequency is detuned relative to the PF central frequency.

There is a need to note the PF bandwidth is matched with the transmitted signal bandwidth. If the detuning value between the PF and AF central frequencies is more than 4 or 5 times the transmitted signal bandwidth to be detected, i.e. $4 \div 5 \Delta f_{s}$, where $\Delta f_{s}$ is the transmitted signal bandwidth, we can believe that the processes at the PF and AF outputs are uncorrelated because the coefficient of correlation between them is negligible (not more than 0.05). This fact was confirmed experimentally in [19] and [20] independently. Thus, the transmitted signal plus noise can be appeared at the GR PF output and the noise only is appeared at the GR AF output. The stochastic processes at the GR AF and GR PF outputs present the input stochastic samples from two independent frequency-time regions. If the discrete-time noise $w_{i}[k]$ at the GR PF and GR AF inputs is Gaussian, the discrete-time noise $\zeta_{i}[k]$ at the GR PF output is Gaussian too, and the reference discrete-time noise $\eta_{i}[k]$ at the GR AF output is Gaussian owing to the fact that the GR PF and GR AF are the linear systems and we believe that these linear systems do not change the statistical parameters of the input process. Thus, the GR AF can be considered as a generator of the reference noise with a priori information a "no" transmitted signal (the reference noise sample) [10, Chapter 5]. The noise at the GR PF and GR AF outputs can be presented as 


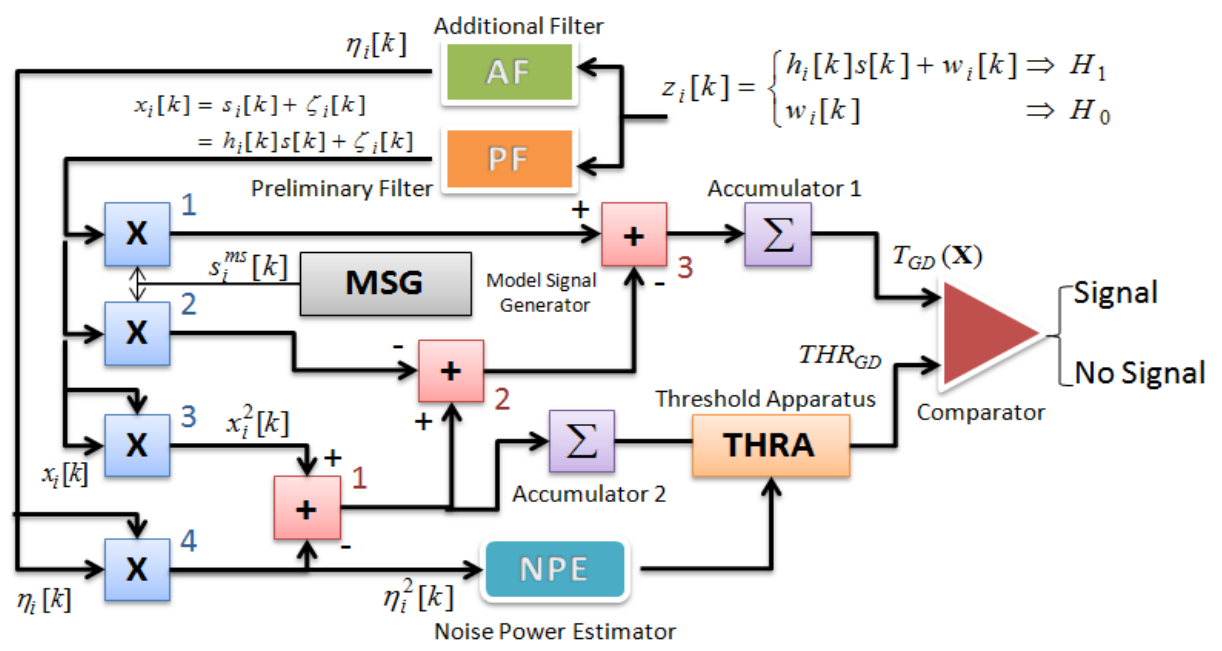

Fig. 3. Generalized receiver.

$$
\left\{\begin{array}{l}
\zeta_{i}[k]=\sum_{m=-\infty}^{\infty} g_{P F}[m] w_{i}[k-m] ; \\
\eta_{i}[k]=\sum_{m=-\infty}^{\infty} g_{A F}[m] w_{i}[k-m],
\end{array}\right.
$$

where $g_{P F}[m]$ and $g_{A F}[m]$ are the impulse responses of the GR PF and GR AF, respectively.

In a general, under practical implementation of any detector in wireless communication system with sensor array, the bandwidth of the spectrum to be sensed is defined. Thus, the GR AF bandwidth and central frequency can be assigned, too (this bandwidth cannot be used by the transmitted signal because it is out of its spectrum). The case when there are interfering signals within the GR AF bandwidth, the action of this interference on the GR detection performance, and the case of non-ideal condition when the noise at the GR PF and GR AF outputs is not the same by statistical parameters are discussed in [21] and [22].

Under the hypothesis $\mathscr{H}_{1}$ ("a yes" transmitted signal), the GR CD generates the signal component $s_{i}^{m}[k] s_{i}[k]$ caused by interaction between the model signal $s_{i}^{m}[k]$, forming at the MSG output, and the incoming signal $s_{i}[k]$, and the noise component $s_{i}^{m}[k]$ $\times \zeta_{i}[k]$ caused by interaction between the model signal $s_{i}^{m}[k]$ and the noise $\zeta_{i}[k]$ at the PF output. GR ED generates the transmitted signal energy $s_{i}^{2}[k]$ and the random component $s_{i}[k] \zeta_{i}[k]$ caused by interaction between the transmitted signal $s_{i}[k]$ and the noise $\zeta_{i}[k]$ at the PF output. The main purpose of the GR CC is to cancel completely in the statistical sen- se the GR CD noise component $s_{i}^{m}[k] \zeta_{i}[k]$ and the GR ED random component $s_{i}[k] \zeta_{i}[k]$ based on the same nature of the noise $\zeta_{i}[k]$. The relation between the transmitted signal to be detected $s_{i}[k]$ and the model signal $s_{i}^{m}[k]$ is defined as:

$$
s_{i}^{m}[k]=\mu s_{i}[k],
$$

where $\mu$ is the coefficient of proportionality.

The main functioning condition under the GR employment in any signal processing system including the communication one with radar sensors is the equality between the parameters of the model signal $s_{i}^{m}[k]$ and the incoming signal $s_{i}[k]$, for example, by amplitude. Under this condition it is possible to cancel completely in the statistical sense the noise component $s_{i}^{m}[k] \zeta_{i}[k]$ of the GR CD and the random component $s_{i}[k] \zeta_{i}[k]$ of the GR ED. Satisfying the GR main functioning condition given by $(22), s_{i}^{m}[k]=$ $s_{i}[k], \mu=1$, we are able to detect the transmitted signal with the high probability of detection at the low SNR and define the transmitted signal parameters with the required high accuracy.

Practical realization of the condition (22) at $\mu \rightarrow$ 1 requires increasing in the complexity of GR structure and, consequently, leads us to increasing in computation cost. For example, there is a need to employ the amplitude tracking system or to use the offline data samples processing. Under the hypothesis $\mathscr{H}_{0}$ ("a no" transmitted signal), satisfying the main GR functioning condition (22) at $\mu \rightarrow 1$ we obtain only the background noise $\eta_{i}^{2}[k]-\zeta_{i}^{2}[k]$ at the GR output. 
Under practical implementation, the real structure of GR depends on specificity of signal processing systems and their applications, for example, the radar sensor systems, adaptive wireless communication systems, cognitive radio systems, satellite communication systems, mobile communication systems and so on. In the present paper, the GR circuitry (Fig.3) is demonstrated with the purpose to explain the main functioning principles. Because of this, the GR flowchart presented in the paper should be considered under this viewpoint. Satisfying the GR main functioning condition (22) at $\mu \rightarrow 1$, the ideal case, for the wireless communication systems with radar sensor applications we are able to detect the transmitted signal with very high probability of detection and define accurately its parameters.

In the present paper, we discuss the GR implementation in the broadband space-time spreading MC DS-CDMA wireless communication system. Since the presented GR test statistics is defined by the signal energy and noise power, the equality between the parameters of the model signal $s_{i}^{m}[k]$ and transmitted signal to be detected $s_{i}[k]$, in particular by amplitude, is required that leads us to high circuitry complexity in practice.

For example, there is a need to employ the amplitude tracking system or off-line data sample processing. Detailed discussion about the main GR functioning principles if there is no a priori information and there is an uncertainty about the parameters of transmitted signal, i. e., the transmitted signal parameters are random, can be found in [9], [10, Chapter 6, pp.611-621 and Chapter 7, pp. 631-695].

The complete matching between the model signal $s_{i}^{m}[k]$ and the incoming signal $s_{i}[k]$, for example by amplitude, is a very hard problem in practice because the incoming signal $s_{i}[k]$ depends on both the fading and the transmitted signal parameters and it is impractical to estimate the fading gain at the low $S N R$. This matching is possible in the ideal case only. The GD detection performance will be deteriorated under mismatching in parameters between the model signal $s_{i}^{m}[k]$ and the transmitted signal $s_{i}[k]$ and the impact of this problem is discussed in [23][26], where a complete analysis about the violation of the main GR functioning requirements is presented. The GR decision statistics requires an estimation of the noise variance $\sigma_{\eta}^{2}$ using the reference noise $\eta_{i}[k]$ at the AF output.

Under the hypothesis $\mathscr{H}_{1}$, the signal at the GR PF output, see Fig. 3, can be defined as

$$
x_{i}[k]=s_{i}[k]+\zeta_{i}[k],
$$

where $\zeta_{i}[k]$ is the noise at the PF output and

$$
s_{i}[k]=h_{i}[k] s[k],
$$

where $h_{i}[k]$ are the channel coefficients. Under the hypothesis $\mathscr{H}_{0}$ and for all $i$ and $k$, the process $x_{i}[k]=$ $\zeta_{i}[k]$ at the PF output is subjected to the complex Gaussian distribution and can be considered as the i.i.d. process.

In the ideal case, we can think that the signal at the GR AF output is the reference noise $\eta_{i}[k]$ with the same statistical parameters as the noise $\zeta_{i}[k]$. In practice, there is a difference between the statistical parameters of the noise $\eta_{i}[k]$ and $\zeta_{i}[k]$. How this difference impacts on the GR detection performance is discussed in detail in [10, Chapter 7, pp. 631-695] and in [23]-[26],

The decision statistics at the GR output presented in [19] and [20, Chapter 3] is extended for the case of antenna array when an adoption of multiple antennas and antenna arrays is effective to mitigate the negative attenuation and fading effects. The GR decision statistics can be presented in the following form:

$$
\begin{gathered}
T_{G R}(\mathbf{X})=\sum_{k=0}^{N-1} \sum_{i=1}^{M} 2 x_{i}[k] s_{i}^{m}[k] \\
-\sum_{k=0}^{N-1} \sum_{i=1}^{M} x_{i}^{2}[k]+\sum_{k=0}^{N-1} \sum_{i=1}^{M} \eta_{i}^{2}[k] \underset{\substack{\text { F }_{0} \\
\underset{F_{1}}{>}}}{>} T H R_{G R},
\end{gathered}
$$

where

$$
\mathbf{X}=[\mathbf{x}(0), \ldots, \mathbf{x}(N-1)]
$$

is the vector of the random process at the GR PF output and $T H R_{G R}$ is the GR detection threshold.

Under the hypotheses $\mathscr{H}_{1}$ and $\mathscr{H}_{0}$ when the amplitude of the transmitted signal is equal to the amplitude of the model signal, $s_{i}^{m}[k]=s_{i}[k], \mu=1$, the GR decision statistics $T_{G D}(\mathbf{X})$ takes the following form in the statistical sense, respectively:

$$
\left\{\begin{array}{l}
\mathscr{H}_{1}: T_{G D}(\mathbf{X})=\sum_{k=0}^{N-1} \sum_{i=1}^{M}\left\{s_{i}^{2}[k]+\eta_{i}^{2}[k]-\zeta_{i}^{2}[k]\right\} \\
\mathscr{H}_{0}: T_{G D}(\mathbf{X})=\sum_{k=0}^{N-1} \sum_{i=1}^{M}\left\{\eta_{i}^{2}[k]-\zeta_{i}^{2}[k]\right\}
\end{array} .\right.
$$

In (27) the term $\sum_{k=0}^{N-1} \sum_{i=1}^{M} s_{i}^{2}[k]=E_{s}$ corresponds to the average transmitted signal energy, and the term $\sum_{k=0}^{N-1} \sum_{i=1}^{M} \eta_{i}^{2}[k]-\sum_{k=0}^{N-1} \sum_{i=1}^{M} \zeta_{i}^{2}[k]$ is the backgr- 
ound noise at the GR output. The GR output background noise is a difference between the noise power at the GR PF and GR AF outputs. Practical implementation of the GR decision statistics requires an estimation of the noise variance $\sigma_{\eta}^{2}$ using the reference noise $\eta_{i}[k]$ at the AF output.

\subsection{Receiver model}

Let the first user be the user of interest and consider a generalized receiver employing the fast Fourier transform (FFT)-based multicarrier demodulation, space-time dispreading, as well as diversity combining, as shown in Fig. 4. The receiver of Fig.4 constructed on the basis of the generalized receiver (Fig. 3) essentially carries out the inverse operations of those seen in Fig. 1. In Fig. 4, the received signal is first demodulated using FFT-based multicarrier demodulation, obtaining $P S$ number of parallel streams corresponding to the signals transmitted on PS subcarriers. Then, each stream is the space-time despread using the approach discussed in [5], [27], and
[28], in order to obtain $G$ separate variables $\left\{Z_{p, 1}\right.$, $\left.Z_{p, 2}, \ldots, Z_{p, G}\right\}_{p=1}^{P S}$ corresponding to the $G$ data bits transmitted on the $p$-th stream, where $p=1,2, \ldots, P S$, respectively.

Following the space-time despreading, a decision variable is formed for each of the transmitted data bits $\left\{b_{p 1}, b_{p 2}, \ldots, b_{p G}\right\}_{p=1}^{P}$ that can be expressed in the following form:

$$
Z_{p i}=\sum_{s=1}^{S} Z_{((s-1) P+p), i}, p=1,2, \ldots, P ; i=1,2, \ldots, G
$$

Finally, the $P G$ number of transmitted data bits can be decided based on the decision variables $\left\{Z_{p i}, p=\right.$ $1,2, \ldots, P ; i=1,2, \ldots, G\}$ using the conventional decision rule of a BPSK scheme. Let us now investigate the achievable $B E R$ performance.

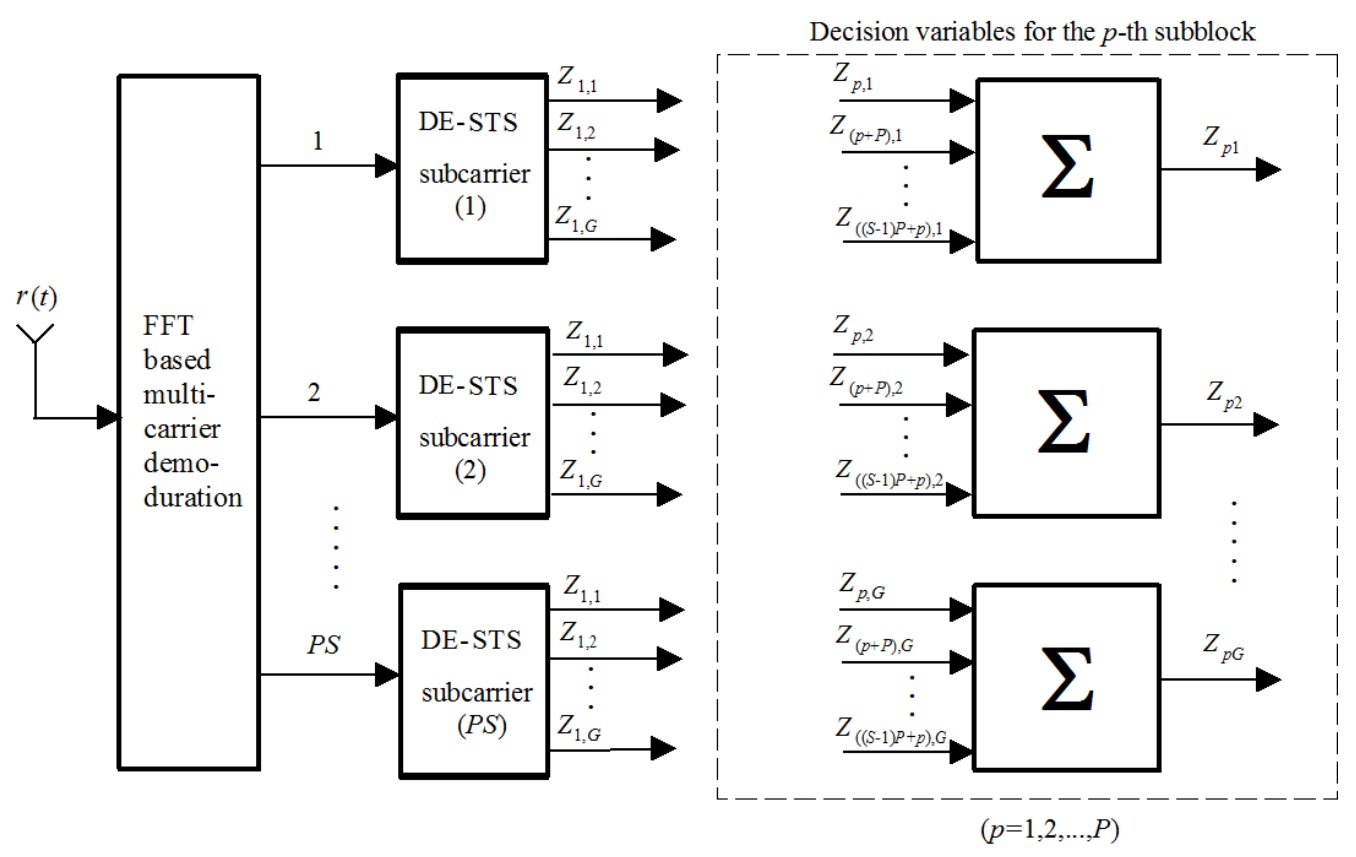

Fig. 4. The receiver schematic of the broadband MC DS-CDMA wireless communication system using space-time spreading.

\section{Bit-Error Rate Analysis}

In this section, we derive the $B E R$ expression of the broadband MC DS-CDMA wireless communication system using the space-time spreading, which was described above in Section II. As an example, we derive the $B E R$ expression in detail for the space-time spreading based on the broadband MC DSCDMA wireless communication system using the following parameters: $G=M=T=2$. The generalized $B E R$

expression of the broadband MC DS-CDMA wireless communication system using the set of attractive space-time spreading schemes, i.e., using $G=M=T=2,4,8$, etc., is then derived from the case $G=M=T=2$ without providing the detailed derivations, since the extension is relatively straightforward. 
In the case $G=M=T=2$, the analysis can be commenced from (20). Let $y_{p, 1}, y_{p, 2}, p=1,2, \ldots, P S$ represent the variables at the output of the generalized detector (Fig. 3) corresponding to the first two data bits transmitted on the $p$-th subcarrier where

$$
\begin{gathered}
y_{p, 1}=\int_{0}^{2 P T_{b}}\left[2 r(t) a_{1,1}(t)-r^{2}(t)+\eta_{1,1}^{2}(t)\right] \\
\times \exp \left(-j 2 \pi f_{p} t\right) d t ; \\
y_{p, 2}=\int_{0}^{2 P T_{b}}\left[2 r(t) a_{1,2}(t)-r^{2}(t)+\eta_{1,2}^{2}(t)\right] \\
\times \exp \left(-j 2 \pi f_{p} t\right) d t .
\end{gathered}
$$

Since the orthogonal multicarrier signals, orthogonal space-time spreading codes, synchronous transmission of the $K$ user signals, as well as slowly flat fading of each subcarrier are assumed, there is no interference between the different users and the different subcarrier signals. Therefore, when substituting (20) into (29) and (30), we obtain

$$
\begin{aligned}
y_{p, 1} & =\frac{4\left(P^{2} T_{b}^{2}-1\right) E_{b}}{P S T_{b}}\left[h_{p 1} \exp \left(j \psi_{p 1}\right) b_{1, p 1}\right. \\
+ & \left.h_{p 2} \exp \left(j \psi_{p 2}\right) b_{1, p 2}\right]+\varsigma_{p, 1} ; \\
y_{p, 2} & =\frac{4\left(P^{2} T_{b}^{2}-1\right) E_{b}}{P S T_{b}}\left[h_{p 1} \exp \left(j \psi_{p 1}\right) b_{1, p 2}\right. \\
& \left.+h_{p 2} \exp \left(j \psi_{p 2}\right) b_{1, p 1}\right]+\varsigma_{p, 2} ;
\end{aligned}
$$

where

$$
\varsigma_{p, i}=\int_{0}^{2 P T_{b}}\left[\eta_{i}^{2}(t)-\xi_{i}^{2}(t)\right] \exp \left(-j 2 \pi f_{p} t\right) d t, i=1,2
$$

that can be approximated by the complex Gaussian variable distributed with the zero mean and variance equal to $8 P T_{b} \mathcal{N}_{0}^{2}$.

Assuming that the generalized receiver has a perfect knowledge with respect to the fading paramet$\operatorname{ers} h_{p i} \times \exp \left(j \psi_{p i}\right), i=1,2$, the decision variables corresponding to the data bits $b_{1, p i}, i=1,2$ associated with the $p$-th subcarrier can be expressed in the following form

$$
\begin{gathered}
Z_{p, 1}=\operatorname{Re}\left\{y_{p, 1} h_{p 1} \exp \left(-j \psi_{p 1}\right)-y_{p, 2} h_{p 2} \exp \left(-j \psi_{p 2}\right)\right\} \\
=\frac{4\left(P^{2} T_{b}^{2}-1\right) E_{b}}{P S T_{b}}\left[h_{p 1}^{2}+h_{p 2}^{2}\right] b_{1, p 1}
\end{gathered}
$$$$
+\operatorname{Re}\left\{\varsigma_{p, 1} h_{p 1} \exp \left(-j \psi_{p 1}\right)-\varsigma_{p, 2} h_{p 2} \exp \left(-j \psi_{p 2}\right)\right\}
$$

$$
\begin{gathered}
Z_{p, 2}=\operatorname{Re}\left\{y_{p, 1} h_{p 2} \exp \left(-j \psi_{p 2}\right)+y_{p, 1} h_{p 1} \exp \left(-j \psi_{p 1}\right)\right\} \\
=\frac{4\left(P^{2} T_{b}^{2}-1\right) E_{b}}{P S T_{b}}\left[h_{p 1}^{2}+h_{p 2}^{2}\right] b_{1, p 2} \\
+\operatorname{Re}\left\{\varsigma_{p, 1} h_{p 2} \exp \left(-j \psi_{p 2}\right)-\varsigma_{p, 2} h_{p 1} \exp \left(-j \psi_{p 1}\right)\right\}(35)
\end{gathered}
$$

where $p=1,2, \ldots, P S$.

Finally, after combining the replicas of the same signal transmitted on the $S$ subcarriers, the decision variables corresponding to the two bits in the $p$-th subblock can be presented in the following form

$$
\begin{aligned}
& Z_{p 1}=\sum_{s=1}^{S} Z_{((s-1) P+p), 1}, \\
& Z_{p 2}=\sum_{s=1}^{S} Z_{((s-1) P+p), 2},
\end{aligned}
$$

where $p=1,2, \ldots, P$.

Since $Z_{((s-1) P+p), 1}$ in (36) and $Z_{((s-1) P+p), 2}$ in (37) are the i.i.d. random variables for different $s$ values associated with the mean

$$
E[Z]=\frac{4\left(P^{2} T_{b}^{2}-1\right) E_{b}}{P S T_{b}}\left[h_{((s-1) P+p) 1}^{2}+h_{((s-1) P+p) 2}^{2}\right]
$$

and the variance

$$
\operatorname{Var}\{Z\}=8 P T_{b} \mathcal{N}_{0}^{\rho 2}\left[h_{((s-1) P+p) 1}^{2}+h_{((s-1) P+p) 2}^{2}\right]
$$

conditioned on $h_{((s-1) P+p) 1}$ and $h_{((s-1) P+p) 2}$, it is well recognized [26], [29] that the conditional $B E R$ can be presented in the following form

$$
B E R\left\{E \mid\left[h_{((s-1) P+p) 1}, h_{((s-1) P+p) 2}\right]\right\}=Q\left(\sqrt{2 \sum_{s=1}^{S} \sum_{g=1}^{2} \gamma_{s g}}\right)
$$

where $Q(x)$ is the Gaussian $Q$-function;

$$
\begin{gathered}
\gamma_{s g}=\bar{\gamma} \times \frac{\left[h_{((s-1) P+p) g}\right]^{2}}{\sigma^{2}} ; \\
\bar{\gamma}=\frac{E_{b} \sigma^{2}}{2 S_{e} \mathcal{N}_{0}^{2}} .
\end{gathered}
$$

Finally, with the aid of (19) and [18], it can be readily shown that the average $B E R$ can be expressed in the following form

$$
B E R=0.5(1-\mu)^{2 S} \sum_{k=0}^{2 S-1}\left(\begin{array}{c}
2 S-1+k \\
k
\end{array}\right) 0.5(1+\mu)^{k},
$$

where 


$$
\mu=\sqrt{\frac{\bar{\gamma}}{1+\bar{\gamma}}} .
$$

In general case $G=M=T=2,4,8, \ldots$, the decision variable $Z_{p, 1}$ corresponding to the first bit in the subblock $p$ can also be expressed as in (36), but with $Z_{((s-1) P+p), 1}$ given by [23]

$$
\begin{aligned}
Z_{((s-1) P+p), 1} & =\frac{4\left(P^{2} T_{b}^{2}-1\right) E_{b}}{P S T_{b}} b_{1, p 1} \sum_{g=1}^{G} h_{((s-1) P+p) g}^{2} \\
& +\operatorname{Re}\left\{\zeta_{((s-1) P+p), 1}^{\prime}\right\}, s=1,2, \ldots, S, \quad
\end{aligned}
$$

where $\operatorname{Re}\left\{\mathcal{S}_{((s-1) P+p), 1}^{\prime}\right\}$ is the background noise with the zero mean and variance equal to

$$
\operatorname{Var}\left\{\operatorname{Re}\left[\zeta_{((s-1) P+p), 1}^{\prime}\right]=8 P T_{b} \mathcal{N}_{0}^{2} \sum_{g=1}^{G} h_{((s-1) P+p) g}^{2} .\right.
$$

The average $B E R$ in the case $G=M=T=2,4,8, \ldots$ can be presented in the following form [24]

$$
B E R=0.5(1-\mu)^{T S} \sum_{k=0}^{T S-1}\left(\begin{array}{c}
2 S-1+k \\
k
\end{array}\right) 0.5(1+\mu)^{k} .
$$

Note that according to (40) and (45) by using the space-time spreading and $F$-domain subcarrier repetition, the diversity order achieved is $T S$ provided that $T$ transmit antennas and $S$-depth $F$-domain repetition schemes were used. The resultant diversity order of $T S$ can also be seen in (47).

Figure 5 demonstrates both the numerical and simulation results of the $B E R$ performance which are drawn using the lines and markers, respectively, at $S=1, T=1,2,4$ as well as at $S=3, T=1,2,4$. From these results we observe that at the $B E R=0.01$ using two transmit antennas rather than one yields a gain of approximately $6.0 \mathrm{~dB}$. Furthermore, when $T=4$ the transmit antennas and the repetition depth $S=3$ are considered instead of $S=1, T=1$, the diversity gain achieved is approximately $9.0 \mathrm{~dB}$.

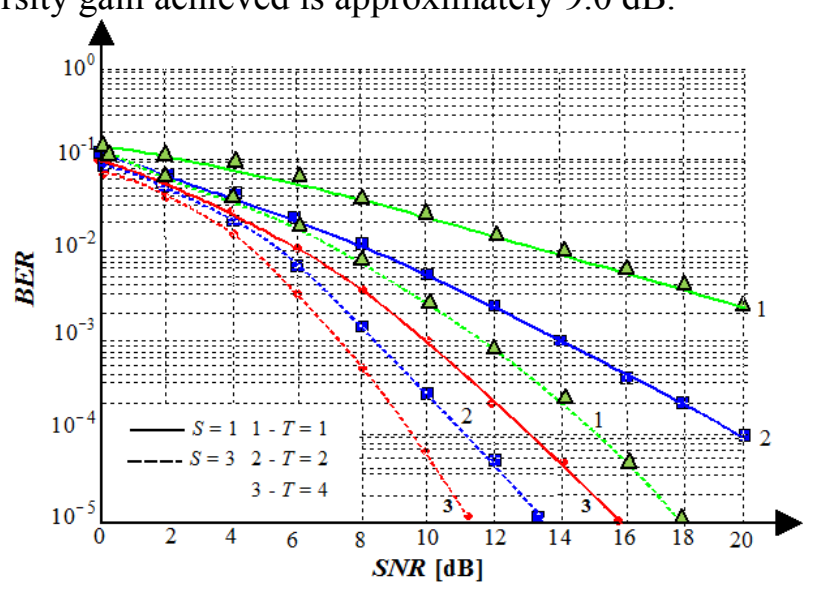

Fig. 5. Numerical (lines) and simulated (markers) $B E R$ versus $S N R$ per bit, $E_{b} / \mathscr{N}_{0}$, performance for the broadband MC DS-CDMA wireless communication system using space-time spreading based on the transmit diversity under the implementation of the generalized receiver when communicating over the frequency-selective Rayleigh fading channels evaluated by (47).

The BER performance presented in Fig.5 can be achieved, provided that the number of orthogonal space-time spreading codes is sufficiently high for supporting the $K$ number of users without reuse. Based on our arguments in Sections II and III, the number of orthogonal space-time spreading codes is given by $P G T_{b} / T_{c}=P G N$. Since each user requires $M$ orthogonal space-time spreading codes, the maximum number of users supported by the $P G N$ orthogonal space-time spreading codes is given by $\kappa_{\text {max }}=$ $P G N / M$.

In other words, if the number of users supported by the synchronous broadband MC DS-CDMA wireless communication system designed according to the philosophy of the present paper obeys $K<K_{\text {max }}$, the $B E R$ performance presented in Fig. 5 can be achieved for transmissions over the frequency-selective Rayleigh fading channels. For the attractive spacetime spreading schemes using $G=M=T=2,4,8, \ldots$, the maximum number of users supported by the orthogonal space-time spreading codes is defined as $K_{\text {max }}=P N$. It can be seen that, in this case, the number of users supported is independent of the number of transmit antennas, which emphasizes the advantages of the space-time spreading schemes using the specific values of $G=M=T=2,4,8, \ldots$ [30].

Let $N=T_{b} / T_{c}$ and the total number of subcarriers $P S$ be constant. Then, the maximum number of users supported by the broadband MC DS-CDMA wireless communication system depends only on the repetition depth $S$. Specifically, the maximum number of users supported decreases when increasing the subcarrier repetition depth $S$, i.e., when increasing the frequency diversity order. Consequently, the maximum number of users supported and the frequency diversity gain achieved have to obey a trade off. This is not a desirable result. We would like to achieve the maximum transmit diversity gain as well as the required frequency diversity gain without having to accept any other trade off, i.e., without decreasing the total number of users supported by the system. Let us now consider this issue in the next section.

\section{Capacity Extension Using Time-}




\section{Frequency Domain Spreading}

\subsection{System description}

In this section, we propose and investigate the broadband MC DS-CDMA wireless communication system, which employs both the time (T)-domain and frequency $(F)$-domain spreading, i.e., it employs $T F$ -domain spreading. The transmitter schematic of the space-time spreading assisted broadband MC DSCDMA wireless communication system using the $T F$-domain spreading is similar to that seen in Fig.1, except that the $S$-depth subcarrier repetition of Fig. 1 is now replaced by the $F$-domain spreading associated with an orthogonal spreading code of length $S$.

Specifically, let $\left\{c_{k}[0], c_{k}[1], \ldots, c_{k}[S-1]\right\}$ be the $k$-th user's orthogonal code in discrete form, which will be used for the $F$-domain spreading. By contrast, the $k$-th user's $T$-domain orthogonal codes used for space-time spreading have been expressed in Section II as $a_{k, i}(t), \quad i=1,2, \ldots, M$ in continuous form. In the broadband MC DS-CDMA wireless communication system using $T F$-domain spreading, the $P$ subblock signals of user $k$ after space-time spreading are now further spread over the $F$-domain using the above $F$-domain spreading codes. The signals transmitted from the $T$ transmit antennas can be presented in the following form

$$
\mathbf{s}_{k}(t)=\operatorname{Re}\left\{\sqrt{\frac{2 E_{b}}{P T_{b} S M T}} \mathbf{D}_{k} \mathbf{Q \omega}\right\},
$$

where all variables have the same interpretations as

those in (6), except that the repetition matrix $\mathbf{P}$ in (6) is now replaced by $\mathbf{Q}$ in (48), which represents the $F$-domain spreading and is the $P \times P S$-dimensional matrix expressed as

$$
\mathbf{Q}=\left\{\mathbf{C}_{k}[0], \mathbf{C}_{k}[1], \ldots, \mathbf{C}_{k}[S-1]\right\},
$$

where $\mathbf{C}_{k}[s], s=0,1, \ldots, S-1$ are the diagonal matrices of rank $P$ which can be presented in the following form

$$
\mathbf{C}_{k}[s]=\operatorname{diag}\left\{c_{k}(s), c_{k}(s), \ldots, c_{k}(s)\right\} .
$$

Specifically, at $G=M=T=2$, the $T F$-domain spread signals transmitted by the transmit antenna 1 and 2 of the broadband MC DS-CDMA wireless communication system can be simply presented in the following form

$$
\mathbf{s}_{k}(t)=\left(\begin{array}{c}
s_{k 1}(t) \\
s_{k 2}(t)
\end{array}\right)=\sqrt{\frac{E_{b}}{2 P T_{b} S}}
$$

$$
\times\left(\begin{array}{l}
\sum_{p=1}^{P} \sum_{s=1}^{S}\left[a_{k, 1} b_{k, p 1}+a_{k, 2} b_{k, p 2}\right] c_{k}(s-1) \cos \left(2 \pi f_{(s-1) P+p} t\right) \\
\sum_{p=1}^{P} \sum_{s=1}^{S}\left[a_{k, 1} b_{k, p 2}-a_{k, 2} b_{k, p 1}\right] c_{k}(s-1) \cos \left(2 \pi f_{(s-1) P+p} t\right)
\end{array}\right)
$$

The total number of users supported by the broadband MC DS-CDMA wireless communication system using the $T F$-domain spreading and the assignment of orthogonal codes to the users is analyzed as follows. According to our analysis in Sections II and III, the total number of orthogonal codes that can be used for the space-time spreading is equal to $P G N$ and the maximum number of users supported by these orthogonal codes is defined as $\%_{\max }=P G N$ $/ M$. By contrast, the total number of orthogonal codes that can be used for $F$-domain spreading is $S$. This implies that even if $S$ that is the number of users shares the same set of space-time spreading codes, these $S$ user signals might be distinguishable with the aid of the associated $S$ number of the $F-$ domain spreading codes.

Explicitly, the total number of users supported is equal to

$$
\Re_{\text {max }}=\frac{S P G N}{M} .
$$

Therefore, the orthogonal spreading codes can be assigned as follows. If the number of users is in the range of $0 \leq K \leq \%_{\max }$, these users will be assigned the required orthogonal space-time spreading codes and the same $F$-domain orthogonal spreading code. However, when the number of users is in the range of $s \%_{\text {max }} \leq K \leq(s+1) \mathcal{K}_{\max }, s=1,2, \ldots, S-1$, then the same set of the $M$ space-time spreading orthogonal codes must be assigned to $s$ or $(s+1)$ users, but these $s$ or $(s+1)$ users are assigned to different $F$-domain spreading codes. These $s$ or $(s+1)$ users employing the same set of space-time spreading orthogonal codes are identified by their corresponding $F$-domain spreading codes.

Since the subcarrier signals across which $F$-domain spreading takes place encounter independent fading, the orthogonality of the $F$-domain spreading codes cannot be retained. Hence, multiuser interference is inevitably introduced that degrades the $B E R$ performance when increasing the number of users sharing the same set of the $M$ space-time spreading orthogonal codes.

Let $1 \leq K^{\prime} \leq S$ be the number of users sharing the same set of space-time spreading orthogonal codes. We also assume that any set of the $M$ space-time spreading orthogonal codes is shared by the same 
$K^{\prime}$ number of users. The, when the $K^{\prime} \times \mathcal{K}_{\max }$ signals expressed in the form of (48) are transmitted over the frequency-selective fading channels, the received complex low-pass equivalent signal can be expressed in the following form

$$
\mathbf{r}(\mathbf{t})=\sum_{k=1}^{K^{\prime} \mathcal{K}_{\max }} \sum_{g=1}^{T} \sqrt{\frac{2 E_{b}}{P T_{b}} \times \frac{1}{S M T}}\left(\mathbf{D}_{k} \mathbf{Q}\right)_{g} \mathbf{H} \boldsymbol{\omega}+\mathbf{w}(\mathbf{t}),
$$

where $\left(\mathbf{D}_{k} \mathbf{Q}\right)_{g}$ and $\mathbf{w}(\mathbf{t})$ have the same interpretation as in (17). Specifically, in the case $G=M=T=2$, $r(t)$ can be expressed in the following form

$$
\begin{aligned}
& r(t)=\sum_{k=1}^{K^{\prime} x_{\max }} \sum_{p=1}^{P} \sum_{s=1}^{S} \sqrt{\frac{2 E_{b}}{4 P S T_{b}}}\left\{h_{(p s) 1} \exp \left(j \psi_{(p s) 1}\right)\right. \\
& \times\left[a_{k, 1}(t) b_{k, p 1}+a_{k, 2}(t) b_{k, p 2}\right] c_{k}(s-1) \\
& +h_{(p s) 2} \exp \left(j \psi_{(p s) 2}\right)\left[a_{k, 1}(t) b_{k, p 2}-a_{k, 2}(t) b_{k, p 1}\right] \\
& \left.\quad \times c_{k}(s-1)\right\} \exp \left(j 2 \pi f_{(s-1) P+p} t\right)+w(t) .
\end{aligned}
$$

The receiver schematic of the broadband MC DS -CDMA wireless communication system using the $T F$-domain spreading is similar to that of Fig. 4, except that the derepetition operation seen in Fig. 4 is now replaced by the $F$-domain despreading. The signals at the output of the space-time-despreading block in Fig. 4 can be detected by invoking a range of single or multiuser detection schemes.

\subsection{Signal detection}

Following the derivations in Section III, in the general case $G=M=T=2,4,8, \ldots$, etc., the decision variable $Z_{((s-1) P+p), 1}$ in terms of the first data bit in the subblock $p$ and the subcarrier $(s-1) P+p$ can now be presented in the following form

$$
\begin{gathered}
Z_{((s-1) P+p), 1}=\frac{4\left(P^{2} T_{b}^{2}-1\right) E_{b}}{P S T_{b}} \sum_{k=1}^{K^{\prime}} \sum_{g=1}^{T} h_{((s-1) P+p) g}^{2} \\
\times c_{k}(s-1) b_{k, p 1}+\operatorname{Re}\left\{\zeta_{((s-1) P+p), i}^{\prime}\right\}, \quad s=1,2, \ldots, S,
\end{gathered}
$$

where $\operatorname{Re}\left\{\varsigma_{p, 1}^{\prime}\right\}$ is the background noise having the zero mean and variance equal to $8 P T_{b} \mathcal{N}_{0}^{2} \sum_{g=1}^{T} h_{p g}^{2}$.

Let

$$
\begin{gathered}
\mathbf{z}_{p 1}=\left[Z_{p, 1} Z_{(P=P), 1} \cdots Z_{((S-1) P+p), 1}\right]^{T}, \\
\mathbf{A}=\operatorname{diag}\left\{\sum_{g=1}^{T_{x}} h_{p g}^{2}, \sum_{g=1}^{T_{x}} h_{(P+p) g}^{2}, \ldots, \sum_{g=1}^{T_{x}} h_{((S-1) P+p) g}^{2}\right\},
\end{gathered}
$$

$$
\mathbf{C}=\left(\begin{array}{cccc}
c_{1}[0] & c_{2}[0] & \cdots & c_{K^{\prime}}[0] \\
c_{1}[1] & c_{2}[1] & \cdots & c_{K^{\prime}}[1] \\
\vdots & \vdots & \ddots & \vdots \\
c_{1}[S-1] & c_{2}[S-1] & \cdots & c_{K^{\prime}}[S-1]
\end{array}\right)
$$

$$
\begin{gathered}
\mathbf{b}=\left[b_{1, p 1} b_{2, p 1} \cdots b_{K^{\prime}, p 1}\right]^{T}, \\
\boldsymbol{\Xi}=\left[\varsigma_{p, 1}^{\prime} \varsigma_{(P+p), 1}^{\prime} \cdots \varsigma_{((S-1) P+p), 1}^{\prime}\right]^{T} .
\end{gathered}
$$

Then Eq. (56) can be written in the following matrix form

$$
\mathbf{z}_{p 1}=\frac{4\left(P^{2} T_{b}^{2}-1\right) E_{b}}{P S T_{b}} \mathbf{A C b}+\mathbf{\Xi}
$$

Detection of signals employed by the broadband MC DS-CDMA wireless communication system given in (62) is similar to the detection of signals employed by the conventional broadband MC DS-CDMA wireless communication system using the $F$-domain spreading, where the single user detectors [6], [7], [21] or multiuser detectors [6], [7], [22]-[24], [30] can be implemented.

In the present paper, we investigate two detection algorithms, namely, the single user generalized detector [23], [24] and the multiuser generalized detector [25], [26] and compare them with the single user correlation detector [6], [7], [15] and the multiuser decorrelating detector [6], [7], [30], [31].

In the context of the generalized detector or receiver, let

$$
\mathbf{z}=\left[Z_{p, 1} Z_{p, 2} \cdots Z_{p, K^{\prime}}\right]^{T}
$$

represents the decision variables. Then, these decision variables are obtained by multiplying both sides of (62) with $\mathbf{C}^{T}$, which can be presented in the following form

$$
\mathbf{z}=\frac{4\left(P^{2} T_{b}^{2}-1\right) E_{b}}{P S T_{b}} \sum_{s=1}^{S} \sum_{g=1}^{T} h_{((s-1) P+p) g}^{2} \times \mathbf{R b}+\mathbf{C}^{T} \boldsymbol{\Xi},
$$

where

$$
\mathbf{R}=\left(\begin{array}{cccc}
1 & \rho_{12} & \cdots & \rho_{1 K^{\prime}} \\
\rho_{21} & 1 & \cdots & \rho_{2 K^{\prime}} \\
\vdots & \vdots & \ddots & \vdots \\
\rho_{K^{\prime} 1} & \rho_{K^{\prime} 2} & \cdots & 1
\end{array}\right)
$$

is the correlation matrix among the $K^{\prime}$ user signals, while $\rho_{i j}$ is the correlation factor between user $i$ and user $j$ that can be presented in the following form 


$$
\rho_{i j}=\frac{\sum_{s=1}^{S}\left\{c_{i}[s-1] c_{j}[s-1] \sum_{g=1}^{T} h_{((s-1) P+p) g}^{2}\right\}}{\sum_{s=1}^{S} \sum_{g=1}^{T} h_{((s-1) P+p) g}^{2}} .
$$

Equation (64) suggests that the diversity gain contributed both by the transmit diversity and frequency diversity can be retained, since we have a double sum of the components $h^{2}$ corresponding to the transmission and frequency diversity orders of $S$ and $T$, respectively. However, the multiuser interference is introduced by the channel's time-varying characteristics. Finally, the corresponding data bits, $b_{k, p 1}, k=$ $1,2, \ldots, K^{\prime}$ are decided according to $\hat{b}_{k, p 1}=\operatorname{sgn} \mathbf{z}_{k}$, $k=1,2, \ldots, K^{\prime}$, where $(\mathbf{z})_{k}$ represents the $k$-th row of $\mathbf{z}$, while sgn( $(\cdot)$ is the sign function [15].

Note that the correlation factors $\left\{\rho_{i j}\right\}$ in (66) are time-variant due to the time-varying nature of the channel's fading envelope. However, since

$$
\sum_{s=1}^{S} c_{i}[s-1] c_{j}[s-1]=0,
$$

it can be shown that $\rho_{i j}=0$, provided that the sum of $\sum_{g=1}^{T} h_{((s-1) P+p) g}^{2}$ is identical for different values of $s$.

Moreover, it can be shown that the correlation factors $\left\{\rho_{i j}\right\}$ are contributed by the differences of the sums $\sum_{g=1}^{T} h_{((s-1) P+p) g}^{2}$ experienced according to the different values of $s$, while the common part of $\sum_{g=1}^{T} h_{((s-1) P+p) g}^{2}$ in terms of different values of $s$ can be successfully removed due to the orthogonality of the $F$-domain spreading codes.

Specifically, let

$$
\sum_{g=1}^{T} h_{((s-1) P+p) g}^{2}=A_{h}+\Delta_{s},
$$

where $A_{h}$ is the average value of $\sum_{g=1}^{T} h_{((s-1) P+p) g}^{2}$ in terms of $s$, while

$$
\Delta_{s}=\sum_{g=1}^{T} h_{((s-1) P+p) g}^{2}-A_{h} .
$$

Then, Eq. (66) can be written as

$$
\rho_{i j}=\frac{\sum_{s=1}^{S}\left\{c_{i}[s-1] c_{j}[s-1] \Delta_{s}\right\}}{\sum_{s=1}^{S}\left(A_{h}+\Delta_{s}\right)} .
$$

Figure 6 demonstrates the probability density function (pdf) of the correlation factor $\rho_{12}$ between the signals of user 1 and user 2 for the two user broadband MC DS-CDMA wireless communication system employing the space-time spreading transmit diversity under communication over the frequency-selective Rayleigh fading channels. The curves in Fig. 6 show that the correlation factor using $T=1,2$ and four transmit antennas is symmetrically distributed around $\rho_{12}=0$

An important observation is that the correlation factor value is predominantly distributed in the vicinty of $\rho_{12}=0$ and becomes similar to the truncated Gaussian random variable distributed within the limits of the interval $[-1,1]$ having a relatively low variance, when increasing the number of transmit antennas. This observation implies that the space-time spreading using several transmit antennas has twofold importance. Firstly, it is capable to provide the spatial diversity. Secondly, it is capable to suppress the multiuser interference imposed by the $F$-domain despreading

In the context of the multiuser generalized detector, the decision variables associated with $\left\{b_{k, p 1}\right\}_{k=1}^{K^{\prime}}$ are obtained by multiplying both sides of (64) with the inverse matrix $\mathbf{R}$, i.e., with the matrix $\mathbf{R}^{-1}$ that can be presented in the following form

$$
\mathbf{R}^{-1} \mathbf{z}=\sqrt{\frac{2 E_{b} P T_{b}}{S}} \sum_{s=1}^{S} \sum_{g=1}^{T} h_{((s-1) P+p) g}^{2} \times \mathbf{b}+\mathbf{R}^{-1} \mathbf{C}^{T} \mathbf{w}
$$

and the corresponding data bits $b_{k, p 1}, k=1,2, \ldots, K^{\prime}$ are decided according to $\hat{b}_{k, p 1}=\operatorname{sgn}\left(\mathbf{R}^{-1} \mathbf{z}\right)_{k}, k=1,2$, $\ldots, K^{\prime}$. Equation (71) shows that each user data can be decided independently of the other user data and the diversity order achieved is TS. Let us now provide a range of simulation results for the space-time spreading broadband MC DS-CDMA wireless communication system using the $T F$-domain spreading. 


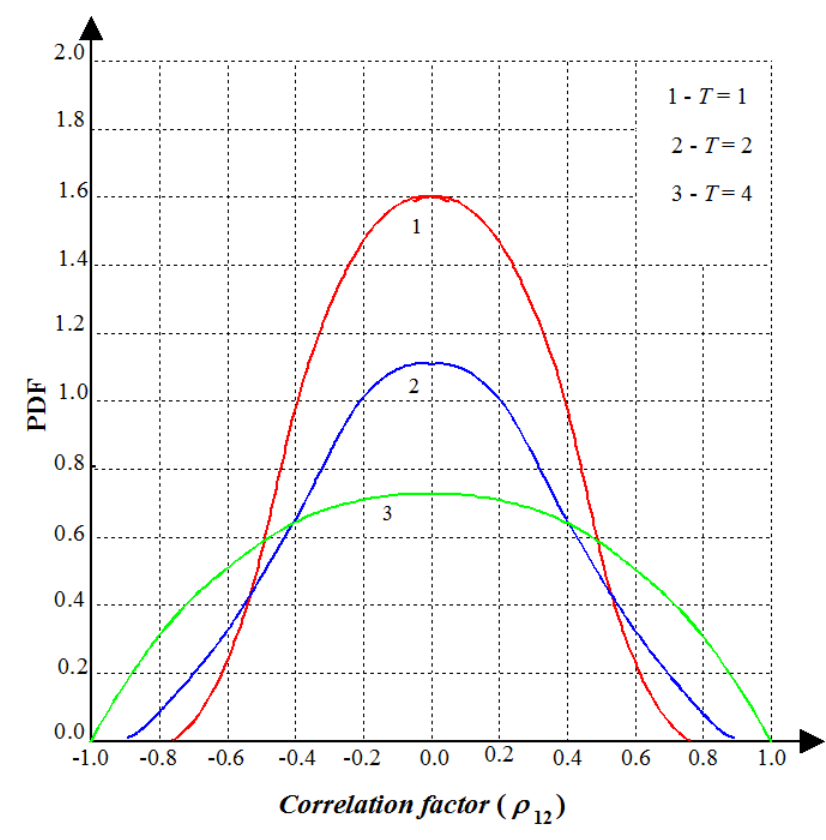

Fig. 6. Simulated pdf of the correlation factor $\rho_{12}$ for the two user broadband MC DS-CDMA communication system using $T=1,2,4$ transmit antennas and the space-time spreading when communicating over the frequency selective Rayleigh fading channels.

\subsection{BER performance}

The $B E R$ versus $S N R$ per bit, $E_{b} / \mathcal{N}_{0}$, performance of the single user and multiuser generalized detector in comparison with both the single user correlation detector and the multiuser decorrelating detector is presented in Figs. 7 and 8 for the broadband $\mathrm{MC}$ DS-CDMA wireless communication system using the $T F$-domain spreading. In both figures we considered $T=2$ transmit antennas and supporting $K=$ $K_{\text {max }}, 2 K_{\text {max }}, 3 \%_{\text {max }}, 4 K_{\text {max }}$ users corresponding to $K^{\prime}=$ $1,2,3,4$, where $\mathscr{K}_{\max }$ represented the maximum number of users supported by the $T$-domain orthogonal spreading codes without imposing the multiuser interference. The condition $K^{\prime}=1$ corresponds to an absence of the multiuser interference. The difference between the Figs. 7 and 8 is that in Fig. 7 the length of the $F$-domain spreading codes is $S=4$, while in Fig. 8 it is $S=8$.

As expected, we observe in both figures that the $B E R$ performance is significantly improved when both the correlation detector and the decorrelating detector are replaced by the generalized detector. For the generalized detector, correlation detector and the decorrelating detector the $B E R$ performance degrades with increasing the number of users sharing the same $T$-domain spreading code, i.e., with increasing the value of $K^{\prime}$. However, the $B E R$ increase due to increasing the value of $K^{\prime}$ is significantly lower for the generalized detector in comparison with the correlation detector and correlating detector Furthermore, upon comparing Figs. 7 and 8, we observe that the $B E R$ performance of the generalized

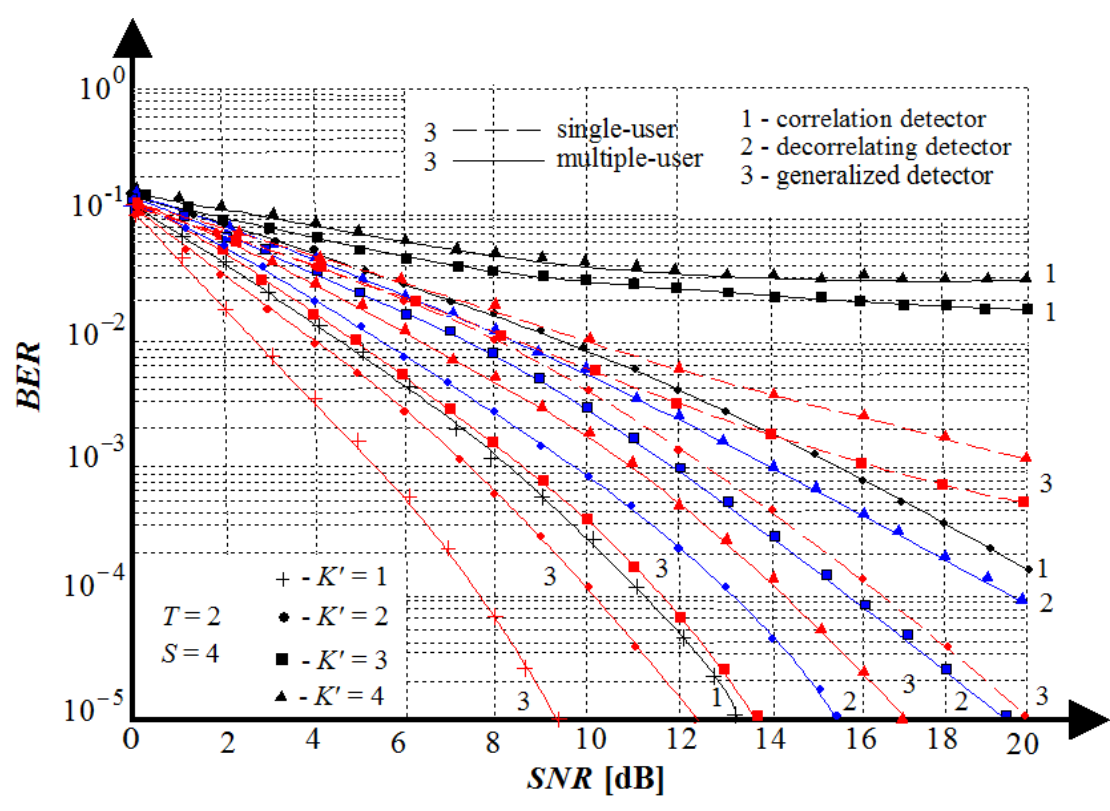

Fig. 7. Simulation of the $B E R$ versus the $S N R$ per bit, $E_{b} / \mathcal{N}_{0}$, performance of the correlation detector, decorrelating detector and generalized detector for the space-time spreading broadband MC DC-CDMA using $T F$-domain spreading when communicating over the frequency-selective Rayleigh fading channels. 


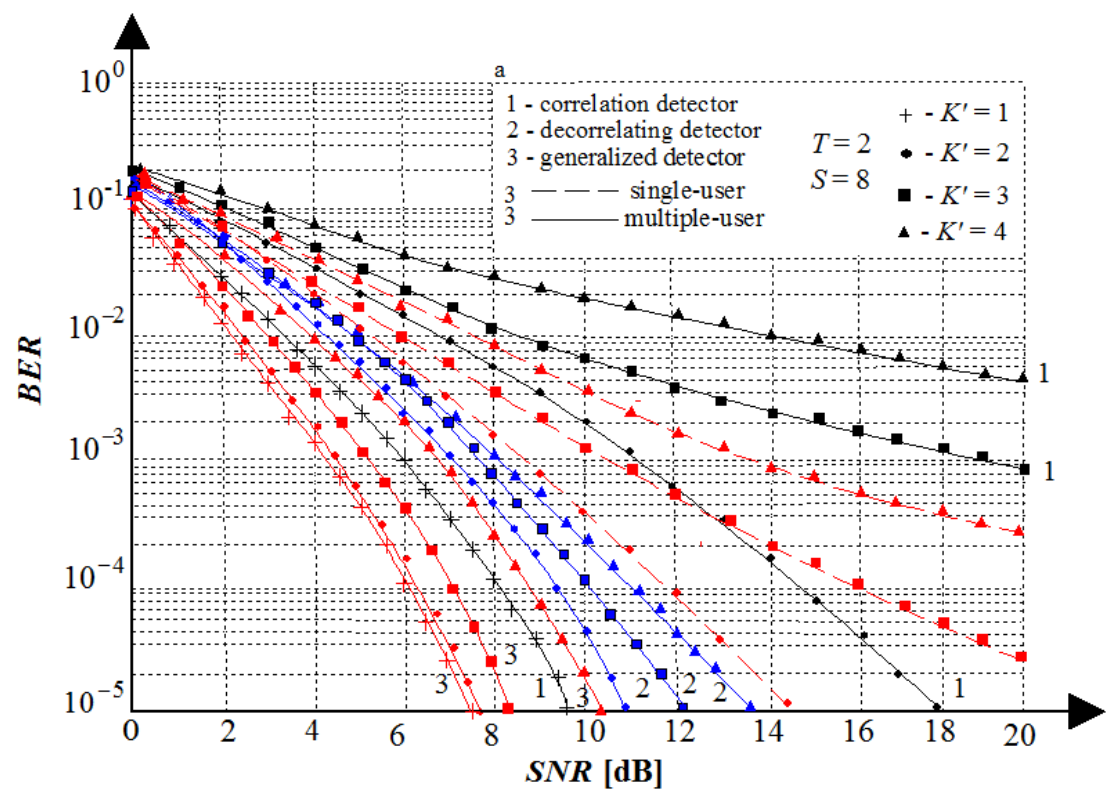

Fig. 8. Simulation of the $B E R$ versus the $S N R$ per bit, $E_{b} / \mathcal{N}_{0}$, performance of the correlation detector, decorrelating detector and generalized detector for the space-time spreading based broadband MC DC-CDMA using $T F$-domain spreading when communicating over the frequency-selective Rayleigh fading channels.

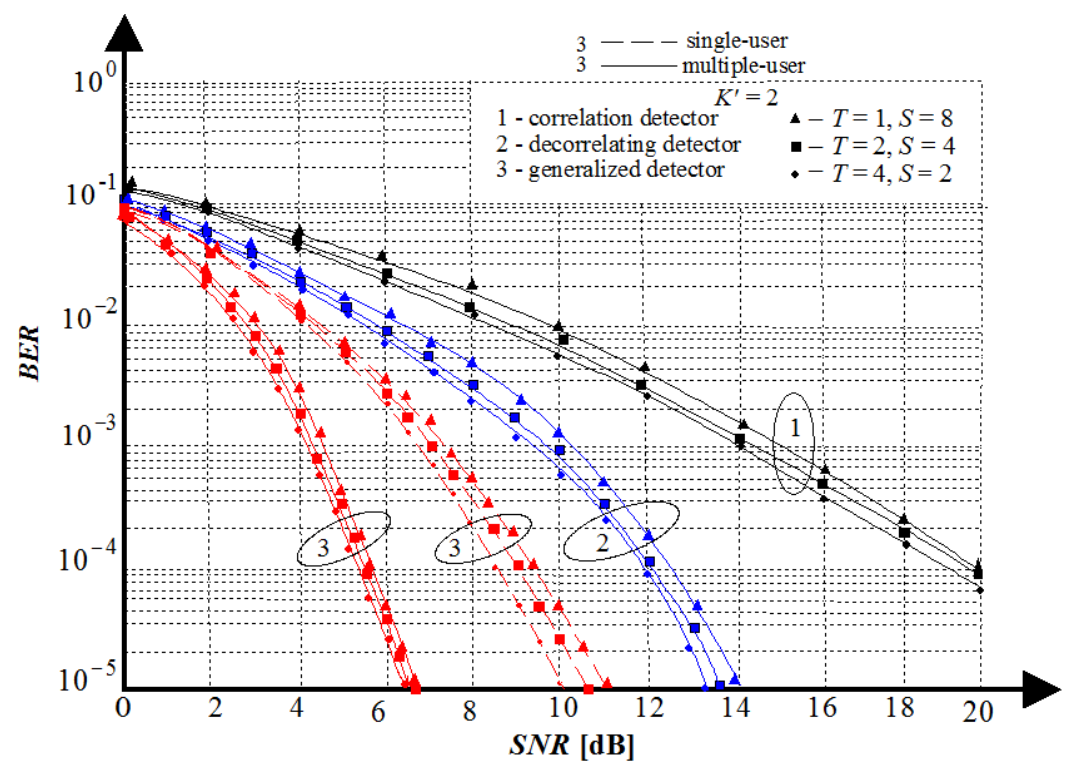

Fig. 9. Simulation of the $B E R$ versus the $S N R$ per bit, $E_{b} / \mathcal{N}_{0}$, performance of the correlation detector, decorrelating detector and generalized detector for the space-time spreading based broadband MC DC-CDMA using $T F$-domain spreading when communicating over the frequency-selective Rayleigh fading channels.

detector is closer to the $B E R$ performance without the multiuser interference, when using $S=8$ (Fig.8) instead of $S=4$ (Fig.7).

In Fig. 9 we investigated the BER performance of the generalized detector in comparison with both the correlation detector and the decorrelating detector at various numbers of the transmit antennas, namely, at $T=1,2,4$ and $S=8,4,2 \quad F$-domain spreading codes, while maintaining a constant $T S$ value of eight. In our experiments, we assumed that $K^{\prime}=2$, i.e., that each set of $T$-domain space-time spreading codes was shared by two users. Let the maximum number of users supported by the $T$-domain spreading codes be $\mathcal{K}_{\max }$, while using the parameters of $T=4, S=2$.

Then for the broadband MC DS-CDMA wireless communication system having a constant system bandwidth, the maximum number of users, $\chi_{\max }$ supported by the $T$-domain codes and using the parameters $T=1, S=8$ or $T=2, S=4$ is $0.25 \%_{\max }$ or $0.5 \%_{\max }$, 
respectively. In other words, there is a maximum of two, four or eight users sharing the same set of the orthogonal space-time spreading codes, corresponding to the cases of $T=4, S=2 ; T=2, S=4$ or $T=1$, $S=8$, respectively.

From the results we infer the following observations:

- All schemes achieve the same total diversity gain,

- The number of transmit antennas has the same effect on the BER performance as the length of the $F$-domain spreading codes, i.e., the same $B E R$ can be maintained, regardless of what values of $T$ and $S$ must be taken to provide that the product $T S$ could be a constant.

- The generalized detector significantly outperforms the correlation detector and decorrelating detector. The gain achieved at the $B E R$ equal to $10^{-3}$ by using the multiuser generalized detector is 6-7 dB in comparison with the multiuser decorrelating detector and by using the single-user generalized detector is $8-9 \mathrm{~dB}$ in comparison with the correlation detector.

- The maximum number of users $K_{\max }^{\prime}$ sharing the same set of space-time spreading orthogonal codes is two, four or eight, when we use the parameters $T=4, S=2 ; T=2$, $S=4 ; T=1, S=8$.

Furthermore, since, according to Figs 7 and 8 , the $B E R$ performance degrades upon increasing the number of users sharing the same set of the space-time spreading orthogonal codes, consequently, for a fully loaded system using the maximum values of $K_{\max }^{\prime}$, we can surmise that the broadband MC DSCDMA wireless communication system employing the parameters $T=4, S=2$ outperforms the one used the parameter combinations $T=2, S=4$ and $T=1$, $S=8$.

Furthermore, the broadband MC DS-CDMA wireless communication system using the parameters $T=2, S=4$ outperforms that employing the parameters $T=1, S=8$. The above arguments suggest that the best broadband MC DS-CDMA wireless communication system will only use the transmit diversity and no frequency diversity at all, i.e., use the parameters $T=8, S=1$, which simultaneously suggests that no multiuser detection is required.

\section{Conclusions}

In the present paper, we have investigated the perfo- rmance of the broadband MC DS-CDMA wireless communication system using the space-time spreading assisted transmit diversity when the frequencyselective Rayleigh fading channels are considered. The issue of parameter design has been investigated motivated by the objective to ensure the same broadband MC DS-CDMA wireless communication system is equipped to provide efficient communications in various fading channels having different grade of frequency selectivity. The BER performance of the broadband MC DS-CDMA wireless communication system using the space-time spreading has been evaluated both analytically and by simulation.

Furthermore, we have considered the capacity extension achievable by the space-time spreading assisted broadband MC DS-CDMA wireless communication system with the aid of $T F$-domain spreading. The corresponding BER performance has been investigated in the context of the multiuser and single user generalized detectors, single user correlation detector and multiuser decorrelating detector for transmissions over the frequency-selective Rayleigh fading channels. In summary, the broadband MC DS -CDMA wireless communication system using space-time spreading assisted transmit diversity has the following characteristics.

- By appropriately selecting the system parameters, the same broadband MC DS-CDMA wireless communication system using the space-time spreading-assisted transmit diversity is rendered capable to achieve the similar $B E R$ performance in various communication environments characterized by different grades of frequency selectivity.

- It is capable to mitigate the peak-to-average power fluctuation experienced, since with the advent of direct sequence spreading of the subcarriers we require only a decreased number of subcarriers.

- Using multiple transmit antennas in the broadband MC DS-CDMA wireless communication system becomes capable to provide the transmit diversity and, simultaneously, suppress the multiuser interference. The associated space-time spreading transmit diversity scheme used in the broadband $\mathrm{MC}$ DS-CDMA wireless communication system can be designed to maintain the constant diversity gain in various fading channels having a different grade of frequency selectivity.

- When the space-time spreading assisted broadband MC DS-CDMA wireless communication system employs both the $T$-domain spreading and the $F$-domain spreading, the 
maximum number of users supported becomes significantly higher than that employing solely the $T$-domain spreading. The higher number of users supported is achieved without any trade off imposed on the achievable diversity order.

\section{References}

[1] V. Tarokh, N. Seshadri, and A.R. Calderbank, Space-time codes for high data rate wireless communication: performance criterion and code construction. IEEE Transactions on Information Theory.1998, Vol. 44, No.2, pp. 744-765.

[2] S.M. Alamouti, A simple transmit diversity technique for wireless communications. IEEE Journal on Selected Areas in Communications. 1998, Vol. 16, No.8, pp. 1451-1458.

[3] L. Hanzo, T.H. Liew, and B.L. Yeap, Turbo Equalization and Space-Time Coding. New York: Wiley \& Sons Inc., 2002

[4] L.-L. Yang and L. Hanzo, Performance of broadband multicarrier DS-CDMA using space-time spreading-assisted transmit diversity. IEEE Transactions on Wireless Communications. 2005, Vol.4, No.3, pp. 885-894.

[5] B. Hochwald, T.L. Marzetta, and C.B. Papadias, A transmitter diversity scheme for wideband CDMA systems based on space-time spreading. IEEE Journal on Selected Areas of Communications. 2001, Vol. 19, No.1, pp. 48-60.

[6] L. Hanzo, L.-L. Yang, E.-L. Kuan, K. Yen, Single- and Multi-Carrier DS-CDMA: Multi-User Detection, Space-Time Spreading, Synchronization, Standards and Networking. New York: IEEE Press/Wiley, 2003.

[7] L.-L. Yang and L. Hanzo, Adaptive space-time spreading-assisted wideband CDMA systems communicating over dispersive Nakagami- $m$ fading channels. EURASIP Journal on Wireless Communications and Networking. 2005, Article \#364197 (2005);doi:10.1155/WCN.2005.216

[8] Telecomm. Industry Association (TIA), TIA/ EIA Interim Standard: Physical Layer Standard for CDMA2000 Standards for Spread Spectrum Systems, 2000

[9] V. Tuzlukov, A new approach to signal detection theory. Digital Signal Processing: Review Journal. 1998, Vol.8, No. 3, pp.166-184.

[10] V. Tuzlukov, Signal Detection Theory. Springer-Verlag, New York, USA, 2001, 746 pp.

[11] V. Tuzlukov, Signal Processing Noise. CRC Press, Boca Raton, London, New York, Washington, D.C., USA, 2002, 692 pp.

[12] L.-L. Yang and L. Hanzo, Performance of ge- neralized multicarrier CDMA in a multipath fading channels. IEEE Transactions on Communications. 2005, Vol.4, No.3, pp. 885-894.

[13] E.A. Sourour and M. Nakagawa, Performance of orthogonal multicarrier CDMA in a multipath fading channels. IEEE Transactions on Communications. 2005, Vol.4, No.3, pp. 356367.

[14] S. Kondo and L.B. Milstein, Performance of multicarrier DS CDMA systems. IEEE Transactions on Communications. 1996, Vol.44, No.2, pp. 238-246.

[15] S. Verdu, Multiuser Detection. Cambridge, U. K.: Cambridge Univ. Press. 1998

[16] Proakis, J.G., Salehi, M. Digital Communications. $5^{\text {th }}$ Edition. New York: McGraw-Hill, USA. 2007, 1150 pages.

[17] J. Feng, X. Zhao, Asymptotic performance analysis of free-space optical links with transmit diversity. Journal of the Optical Society of Korea. 2016, Vol. 20, Issue 4, pp. 451-463.

[18] M. Wen, Z. Beixiong, K.J. Kim, M. Di Renzo, T.A. Tsiftsis, K.-C. Chen, N. Al-Dahir, A survey on spatial modulation in emerging wireless systems: research progress and applications. IEEE Journal on Selected Areas in Communications. 2019, TR 2019-112, pages 22.

[19] M. Maximov, Joint correlation of fluctuative noise at outputs of frequency filters. Radio Engineering. 1956, No. 9, pp. 28-38.

[20] Y. Chernyak, Joint correlation of noise voltage at outputs of amplifiers with no overlapping responses. Radio Physics and Electronics. 1960, No. 4, pp. 551-561.

[21] Shbat, M., Tuzlukov, V.P. Definition of adaptive detection threshold under employment of the generalized detector in radar sensor systems. IET Signal Processing. 2014, Vol. 8, Issue 6, pp. 622-632.

[22] Tuzlukov, V.P. DS-CDMA downlink systems with fading channel employing the generalized. Digital Signal Processing. 2011. Vol. 21, No. 6, pp. 725-733.

[23] Communication Systems: New Research, Editor: Vyacheslav Tuzlukov, NOVA Science Publishers, Inc., New York, USA, 2013, 423 pp.

[24] Shbat, M., Tuzlukov, V.P. Primary signal detection algorithms for spectrum sensing at low SNR over fading channels in cognitive radio. Digital Signal Processing (2019). https://doi. org/10.1016/j.dsp. 2019.07.16. Digital Signal Processing. 2019, Vol. 93, No. 5, pp. 187- 207.

[25] V. Tuzlukov, Signal processing by generalized receiver in wireless communications systems over fading channels," Chapter 2 in Advances 
in Signal Processing. IFSA Publishing Corp. Barcelona, Spain. 2021. pp. 55-111.

[26] V. Tuzlukov, Interference Cancellation for MIMO Systems Employing the Generalized Receiver with High Spectral Efficiency". WSEAS Transactions on Signal Processing. 2021, Vol. 17, Article \#1, pp. 1-15.

[27] N.Minallah, K. Ullah, J. Fruda,K. Cengiz, M. A. Javed, Transmit diversity gain technique aided irregular channel coding for mobile video transmission. Entropy (Basel). 2021, Vol. 23, No.2, pp. 235-243.

[28] N. Minallah,I. Ahmed, M. Ijaz, A.S. Khan, L. Hasan, A. Rehman, On the performance of self -concatenated coding for wireless mobile video transmission using DSTS-SP-assisted smart antenna system. Wireless Communications and Mobile Computing. Vol. 2021, Article ID 8836808,10 pages, 2021. DOI:10.1155/2021/ 8836808 .

[29] M. Shbat and V. Tuzlukov, SNR wall effect alleviation by generalized detector employment in cognitive radio networks. Sensors, 2015, 15 (7), pp.16105-16135;doi:10.3390/s150716105.

[30] V. Tuzlukov, Signal processing by generalized detector in DS-CDMA wireless communication systems with frequency-selective channels. $\mathrm{Ci}$ rcuits, Systems, and Signal Processing. Published on-line on February 2, 2011, doi:10.1007/ s00034-011-9273-1; 2011, Vol.30, No.6, pp. 1197-1230.

[31] M. Shbat, V. Tuzlukov, Generalized detector as a spectrum sensor in cognitive radio networks, "Radioengineering , 2015, Vol. 24, No. 2, pp. 558-571.

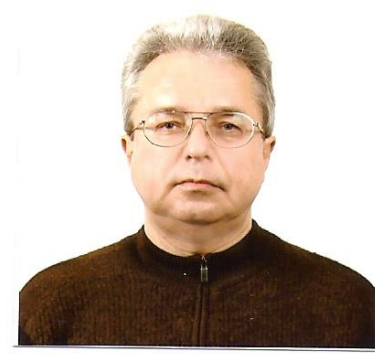
the Belarusian State University, Minsk, Belarus in 1976 and 1990, respectively, and DSc degree in radio physics from the Kotelnikov Institute of Radioengineering and Electronics of Russian Academy of Sciences in 1995. Starting from 1995 and till 1998 Dr. Tuzlukov was a Visiting Professor at the University of San-Diego, San-Diego, California, USA. In 1998 Dr. Tuzlukov relocated to Adelaide, South Australia, where he served as a Visiting Professor at the University of Adelaide till 2000. From 2000 to 2002 he was a Visiting Professor at the University of Aizu, Aizu-Wakamatsu City, Fukushima, Japan and from 2003 to 2007 served as an Invited Professor at the Ajou University, Suwon, South Korea, within the Department of Electrical and Computer Engineering. Starting from March 2008 to February 2009 he joined as a Full Professor at the Yeungnam University, Gyeonsang, South Korea within the School of Electronic Engineering, Communication Engineering, and Computer Science. Starting from March 1, 2009 Dr. Tuzlukov served as Full Professor and Director of Signal Processing Lab at the Department of Communication and Information Technologies, School of Electronics Engineering, College of IT Engineering, Kyungpook National University, Daegu, South Korea. Currently, Dr. Tuzlukov is the Head of Department of Technical Exploitation of Aviation and Radio Engineering Equipment, Belarusian State Academy of Aviation, Minsk, Belarus. His research emphasis is on signal processing in radar, wireless communications, wireless sensor networks, remote sensing, sonar, satellite communications, mobile communications, and other signal processing systems. He is the author over 280 journal and conference papers, seventeenth books in signal processing published by Springer-Verlag and CRC Press. Some of them are Signal Detection Theory (2001), Signal Processing Noise (2002), Signal and Image Processing in Navigational Systems (2005), Signal Processing in Radar Systems (2012), Editor of the book Communication Systems: New Research (2013), Nova Science Publishers, Inc, USA, and has also contributed Chapters "Underwater Acoustical Signal Processing" and "Satellite Communications Systems: Applications" to Electrical Engineering Handbook: $3^{\text {rd }}$ Edition, 2005, CRC Press; "Generalized Approach to Signal Processing in Wireless Communications: The Main Aspects and Some Examples" to Wireless Communications and Networks: Recent Advances, InTech, 2012; "Radar Sensor Detectors for Vehicle Safety Systems" to Electrical and Hybrid Vehicles: Advanced Systems, Automotive Technologies, and Environmental and Social Implications, Nova Science Publishers, Inc., USA, 2014; "Wireless Communications: Generalized Approach to Signal Processing" and "Radio Resource Management and Femtocell Employment in LTE Networks", to Communication Systems: New Research, Nova Science Publishers, Inc., USA, 2013; "Radar Sensor Detectors for Vehicle Safety Systems" to Autonomous Vehicles: Intelligent Transport Systems and Automotive Technologies, Publishing House, University of Pitesti, Romania, 2013; "Radar Sensor Detectors for Vehicle Safety Systems," to Autonomous Vehicles: Intelligent Transport Systems and Smart Technologies, Nova Science Publishers, Inc., New York, USA, 2014; "Signal Processing by Generalized Receiver in DS-CDMA Wireless Communication Systems," to Contemporary Issues in Wireless Communications. INTECH, CROATIA, 2014; "Detection of Spatially Distributed Signals by Generalized Receiver Using Radar Sensor Array in Wireless Communications," to Advances in Communications and Media Research. NOVA Science Publishers, Inc., New York, USA, 2015; "Signal Processing by Generalized Receiver in Wireless Communications Systems over Fading Channels" to Advances in Sig- 
nal Processing. IFSA Publishing Corp. Barcelona, Spain. 2021.

He participates as the General Chair, Keynote Speaker, Plenary Lecturer, Chair of Sessions, Tutorial Instructor and organizes Special Sections at the major International Conferences and Symposia on signal processing.

Dr. Tuzlukov was highly recommended by U.S. experts of Defence Research and Engineering (DDR\& E) of the United States Department of Defence as a recognized expert in the field of humanitarian demining and minefield sensing technologies and had been awarded by Special Prize of the United States Department of Defence in 1999 Dr. Tuzlukov is distinguished as one of the leading achievers from around the world by Marquis Who's Who and his name and biography have been included in the Who's Who in the World, 2006-2013; Who's Who in World, $25^{\text {th }}$ Silver Anniversary Edition, 2008, Marquis Publisher, NJ, USA; Who's Who in Science and Engineering, 2006-2012 and Who's Who in Science and Engineering, 10th Anniversary Edition, 2008-2009, Marquis Publisher, NJ, USA; 2009-2010 Princeton Premier Business Leaders and Professionals Honours Edition, Princeton Premier Publisher, NY, USA; 2009 Strathmore's Who's Who Edi- tion, Strathmore's Who's Who Publisher, NY, USA; 2009 Presidental Who's Who Edition, Presidental Who's Who Publisher, NY, USA; Who's Who among Executives and Professionals, 2010 Edition, Marquis Publisher, NJ, USA; Who's Who in Asia 2012, $2^{\text {nd }}$ Edition, Marquis Publisher, NJ, USA; Top 100 Executives of 2013 Magazine, Super Network Publisher, New York, USA, 2013; 2013/2014 Edition of the Global Professional Network, Business Network Publisher, New York, USA, 2013; 2013/2014 Edition of the Who's Who Network Online, Business Network Publisher, New York, USA, 2014; Online Professional Gateway, 2014 Edition, Business Network Publisher, New York, USA, 2014; 2014 Worldwide Who's Who", Marquis Publisher, NJ, USA; 2015 Strathmore Professional Biographies, Strathmore's Who's Who Publisher, NY, USA; Who's Who in World, 2015, Marquis Publisher, NJ, USA; 2015-2016 Membership in Exclusive Top 100 network of professionals in the world, NY, USA, 2015; 2015 Who's Who of Executives and Professionals Honors Edition, Marquis Publisher, NJ, USA; Worldwide Who's Who - Top 100 Business Networking, San Diego, CA, USA, 2015.

Phone: +375173453283

\section{Creative Commons Attribution License 4.0} (Attribution 4.0 International, CC BY 4.0)

This article is published under the terms of the Creative Commons Attribution License 4.0 https://creativecommons.org/licenses/by/4.0/deed.en US 